\title{
A study on the stress gradient reconstruction in finite elements problems with application of radial basis function networks
}

\author{
Giorgio Previati · Massimiliano Gobbi · Federico Ballo
}

Received: date / Accepted: date

\begin{abstract}
The recovery of the stress gradient in finite elements problems is a widely discussed topic with many applications in the design process. The stress gradient is related to the second derivative (Hessian) of the nodal displacements and numerical techniques are required for its calculation. Particular difficulties are encountered in the reconstruction of the stress gradient in the boundary regions of the domain. This is of particular concern in most applications, especially in mechanical components, where the maximum values of stresses are often located in these regions and the stress gradient has a strong influence on the fatigue life of the component.

This paper presents a comparison between some already published, partially modified, recovery techniques and a different approach based on radial basis function networks. The aim of the paper is to compare the performances of the different approaches for a number of element types with particular focus on the boundary regions. Some examples of mechanical interest are considered.
\end{abstract}

\section{Introduction}

Finite elements analysis is a standard tool for the solution of many problems in engineering. Finite element codes can solve mathematical model based on partial differential equations that cannot be solved in analytical form [12].

Department of Mechanical Engineering,

Politecnico di Milano,

Via La Masa, 1, 20156, Milan, Italy

Tel.: +39-02-23998606

E-mail: federicomaria.ballo@polimi.it
Models based on standard $C^{0}$ elements can provide piecewise continuous approximation of the unknown solution of the model. First order derivative of the solution can be computed with a good level of accuracy only in given points of the elements [55] and are discontinuous across different elements [57]. Second order derivatives cannot be computed if linear or bi-linear (from now on denoted as linear for the sake of simplicity) elements are used and are very poorly approximated with quadratic elements.

The computation of first (gradient) and second derivative (hessian) of the solution field of finite element problems are well discussed topics. The use of $C^{1}$ or mixed $C^{0-1}$ continuity elements can provide continuous derivatives across the elements $[50,40,51]$. Such implementations require additional degrees of freedom [17] at nodes leading to high computational costs. Moreover, additional boundary conditions are required [51, 9, 34]. The computation of such boundary condition is still not completely solved and these elements are still under development $[7,11]$.

A different approach consists in the a posteriori reconstruction of the first and second derivatives [12]. Recovery methods smooth the derivative fields, are able to extract high order information and can generally be adapted to different applications and numerical schemes [43]. In general, these methods start from some result of the finite element analysis (typically the value of the solution at nodes $[54,19]$ or of its derivative at some particular point $[55,56])$ and by means of interpolation approximate the first or second derivative in some points of interest. The interpolation is performed over a patch of elements. The reconstructed derivatives are continuous inside each patch.

The order of the interpolating polynomial of the gradient field over a patch is usually limited by the num- 
ber of superconvergent points present inside the patch [20]. Superconvergent points are locations inside the element where the solution is more accurate, i.e. they are optimal sampling points in the elements where the raw stresses obtained from the finite element solution show a higher-order accuracy [20]. If interpolating functions of order higher than the order of the shape functions are used, ill-conditioned matrices can result in the patch interpolation. To overcome this problem and improve boundary stress recovery, equilibrium constraint can be included in the patch recovery [20, 2, 24, 5, 48].

Alternative recovery techniques that do not require the knowledge of superconvergent points are the 'posteriori equilibrium method' (PEM) [41], the 'recovery by equilibrium in patches' (REP) [5] and the 'recovery by compatibility in patches' (RCP) [42]. As well explained in [32], all these methods are based on a mixed interpolation approach where the point-wise stress-strain relationship of the displacement-based finite element method is relaxed and applied over the entire element volume using Lagrange multipliers. In the same paper the authors also propose an improved procedure for computing stresses based on the mixed interpolation approach.

If the smoothing operator is applied to the whole model (or large sections of the model) global recovery techniques can be constructed. These approaches are usually more computationally expensive, require more complex recovery algorithms and in the early formulations performed poorly near boundaries [20]. In [35] a global approach with reduced computational cost is presented and shows very interesting performances, in particular a good accuracy in the stress recovery at boundary nodes. However, the implementation in 3d problems especially with quadratic polynomials and elements with inside nodes seems yet to be developed.

The reconstruction of the derivatives finds many applications. In error estimation, the reconstructed and smoothed gradient and Hessian are used to build a error index [12, 33, 43]. Many of these works are focused on adaptive mesh refinement [38]. In plasticity models, the stress gradient plays an important role in the computation of the elasto-plastic behaviour of the materials. In these models, reconstruction techniques can be used during the solution of the system equations [49].

In mechanical problems, the first derivative of the displacement field is related to the stress computation. The correct computation of the stress field is of great importance, especially in boundary regions. The computation of the stress gradient (related to the Hessian of the displacement field) is also important for fatigue life assessment, where the stress gradient effect in fatigue life is increasingly investigated [31], computation of the plasticity deformations [15, 25] and Hydrogen embitterment [25, 44].

The essence of the FE method is that a problem domain can be divided into not overlapping elements. The considered field function (displacement, temperature, velocity...) is approximated within the elements through simple interpolation functions. The recovery of function gradients and Hessians can be seen from a mathematical point of view as a problem of interpolation. Given that the primary solution of a finite element problem is best approximated at nodes and gradients at Gauss points, starting from these points other interpolation techniques can be investigated. Being different from element-based technique, meshless methods approximate field functions within an influence domain instead of an element. Different influence domains may overlap. Meshless methods have been achieved remarkable progress in recent years. A point interpolation meshless method based on radial basis function networks (RBFN) has been applied in [46]. Particularly, Gaussian and multiquadric radial basis functions have been considered in [8].

This paper is mainly concerned with mechanical problems, so the first derivative recovery will be referred to as stress recovery and the second derivative recovery as stress gradient recovery. However, the considerations reported in the paper are of general applicability respectively for gradient and hessian recovery in finite element problems.

The paper is organized as follows. Firstly some stress and stress gradient recovery techniques are presented. The techniques have been chosen, among the many available in the literature, on the base of previous published performances and simplicity of implementation. Also, one stress recovery and one stress gradient recovery technique implemented in commercial finite element software are considered. Then, RBFN are introduced and applied to the recovery problems. Finally, two numerical examples, of mechanical interest, one in two dimensions and one in three dimensions, are presented and discussed.

\section{Stress recovery techniques}

In this section the stress recovery techniques considered in the paper are described. Three techniques have been chosen, namely the nodal average, the ZZ patch recovery and the polynomial preserving recovery. The first one is implemented in most commercial finite element software and deserves an analysis of its performances. The other two are the most used techniques and have proved to be a reliable tool. All these techniques are 
also the basis for the stress gradient recovery techniques considered in the next section.

Before defining the stress recovery techniques, let us consider the following notation.

$\Omega \quad$ Region of the considered space $\mathbb{R}^{2}$ or $\mathbb{R}^{3}$

$\partial \Omega \quad$ Frontier of $\Omega$

$i \quad$ Index of a generic node

$j \quad$ Index of a vertex, i.e. a node on a vertex of an element but not on $\partial \Omega$

$V_{k} \quad$ Approximation space of order $k$

$v_{k} \quad$ Polynomial function of order $k$

$n_{k} \quad$ Shape function of order $k$

$\sigma_{i} \quad$ Generic stress component at node $i$

$\sigma_{j} \quad$ Generic stress component at node $j$

$\sim \quad$ Smoothing operator

\subsection{Nodal average}

The first recovery method considered is the weighted average of nodal stress values $(W A N S)$ derived by extrapolating the stresses computed at Gauss points [1]. This method is widely employed by finite elements commercial codes $[49,23,39,29,1]$. By calling $\sigma_{i, l}$ the generic component of stress at a given node $i$ from the element $l$ connected at the node, the weighted average stress can be computed as

$\widetilde{\sigma}_{i}=\frac{\sum_{l=1}^{m} \omega_{l} \sigma_{i, l}}{\sum_{l=1}^{m} \omega_{l}}$

where $m$ is the number of elements connected at node $i$, and $\omega_{l}$ are the weights (element areas or volumes). Small differences in the definition of the weights can be found in different implementation of the methods.

\section{$2.2 \mathrm{ZZ}$ patch recovery}

The $Z Z$ patch recovery method $(Z Z P R)$ has been firstly published by Zienkiewicz and Zhu $[55,56]$. The method is based on the definition of the optimal sampling points of the elements, i.e. in the definition of those points in which the FE solution of the stress field is best approximated. For $C^{0}$ elements, these points coincide with the Gauss points of the reduced integration scheme [57] (see [22] for a discussion on superconvergent points). For regular quadrilateral meshes and strongly regular triangular meshes these points are superconvergent [21, 53]. For triangular or irregular meshes, the superconvergent properties cannot be demonstrated analytically but many numerical experiments have shown that at these points the approximation of the stress field is excellent [57]. The values of stress at the optimal sampling points is used to compute the stress value at nodes. The procedure for the computation of the stresses at nodes is the following.

(a) For each node a patch of elements is constructed. For vertex nodes, the patch is constructed by considering all the elements connected to that node. Boundary nodes and edge nodes require a special treatment (see [57] for a detailed discussion on patch assembly).

(b) The value of the stress at the Gauss points of the elements of the patch are interpolated by a polynomial function. The interpolation is performed in the least square sense. Calling $P_{l}$ the $n$ sampling points (i.e. the Gauss points of the elements belonging to the patch), the residual function $R$ reads

$R=\sum_{l=1}^{n}\left(\sigma\left(P_{l}\right)-v_{k}\left(P_{l}\right)\right)^{2}$

where $\sigma\left(P_{l}\right)$ is the generic component of stress evaluated at point $P_{l}$ and $v_{k}\left(P_{l}\right)$ is the value of the interpolating polynomial function at $P_{l}$. Eq. 2 can be minimized in the least square sense by the matricial formula [3]

$\boldsymbol{a}=\boldsymbol{P}^{\dagger} \boldsymbol{b}$

where $\boldsymbol{a}=\left[\begin{array}{llll}a_{1} & a_{2} & \ldots & a_{m}\end{array}\right]^{T}$ is the vector of the $m$ coefficients of the polynomial function $v_{k}$, $\boldsymbol{b}=\left[\sigma\left(P_{1}\right) \sigma\left(P_{2}\right) \ldots \sigma\left(P_{l}\right)\right]^{T}$ is the vector of the stress values at the sampling points, $\boldsymbol{P}$ is the matrix of the coordinates of the sampling points defined as follows and the symbol ${ }^{\dagger}$ represents the pseudoinverse operation [3].

Let us define $\zeta_{k}$ a base of monomial functions from 1 to $x_{r}^{k}$ where $x_{r}$ represents the different coordinates of the point ( $x$ and $y$ for 2D models and $x, y$ and $z$ for 3D models). Also mixed monomials can be considered. The actual composition of the base depends on the choice of the order of the interpolating function. To avoid numerical problems, Zienkiewicz and Zhu suggested that the order of the interpolating polynomial function is the same of the order of the shape function of the element. In Appendix A the expressions of the bases used for different element 
types are reported. Matrix $\boldsymbol{P}$ can be assembled as

$$
\boldsymbol{P}=\left[\begin{array}{c}
\zeta_{k}\left(P_{1}\right) \\
\zeta_{k}\left(P_{2}\right) \\
\vdots \\
\zeta_{k}\left(P_{n}\right)
\end{array}\right]
$$

The pseudo-inverse of $\boldsymbol{P}$ can be defined only if $n \geq$ $m$. If this condition is not satisfied, the number of sampling points has to be increased by adding elements to the patch [43].

(c) The stress at nodes is computed from the interpolating polynomial function. For vertex nodes, the stress value is computed directly from the patch centred on that vertex. For non vertex nodes (i.e. nodes on the edges of the elements or nodes on the vertex of the elements but laying on $\partial \Omega$ ), the stress value is the mean of the stress values computed for that node in all the patches where the node is present.

This recovery methods has been firstly developed for linear elastic problems [55, 57] as error estimation technique and for adaptive meshes. However, it has also been successfully employed for non linear [14] and elasto-plastic problems [15].

The patch recovery technique has been widely applied in the stress recovery and several modifications have been proposed. In particular, the method can be improved by considering weak $([48,4])$ or strong $([37])$ equilibrium conditions. Since the following analysis is focused not only on stress recovery but also on the recovery of the stress gradient, these improvements are not considered in the present paper due to the complexity of applying such conditions to the stress derivatives.

\subsection{Polynomial preserving recovery}

The polynomial preserving recovery method $(P P R)$ has been introduced by Zhang and Naga in $[54,30]$ where they also proved that the method is superconvergent for uniform linear triangular meshes and ultraconvergent (i.e. the convergence rate is two order higher than the optimal global rate [52]) for quadratic regular elements. The method is similar to the ZZ patch recovery in the sense that it interpolates the considered quantities by polynomial functions over a set of sampling points and the coefficients are computed by a least square approach. The main difference is that only nodal values are considered and the displacement field is interpolated instead of the stress field.

An equation formally identical to eq. 3 can be build. In this case, vector $\boldsymbol{b}$ contains nodal displacements $D_{r}$ and reads $\boldsymbol{b}=\left[D_{r}\left(P_{1}\right) D_{r}\left(P_{2}\right) \ldots D_{r}\left(P_{l}\right)\right]^{T}$, with $r=$ $x, y, z$. Matrix $\boldsymbol{P}$ is build in the same way of eq. 4 but the monomials basis are of order $k+1$ (as suggested in [54]) and are reported in Appendix B.

It can be observed that in this method all the nodes are treated in the same way and there is no distinction between vertex or edge nodes. The number of considered nodes must be at lest equal to the number of the coefficients of the interpolating polynomial function. In this paper, the sampling nodes are chosen by considering a circle (or sphere) of radius $\rho$ around the considered node. If the number of nodes at a distance less than $\rho$ is less than a certain threshold, $\rho$ is incremented to include a sufficient number of nodes. The threshold values are chosen for each interpolating function in order to have a relatively large set of nodes and an over-constraint system in eq. 3 and are reported in Appendix B. The choice of large sets can guarantee a stable and accurate solution [43].

The interpolating function is then derived at nodes and the value of the derivative is the approximation of the gradient of the finite element solution at nodes.

The method has been proposed for the recovery of the gradient of a finite element solution, i.e. for a mechanical model where the solution is the displacement, the recovered values correspond to the diagonal components of the strain tensor.

Let us consider a 2D model and call $D_{x}(x, y)$ and $D_{y}(x, y)$ the interpolating functions for the displacements in $x$ and $y$ directions. Let $\widetilde{\boldsymbol{\epsilon}}_{j}$ be the vector containing the three components $\left(\widetilde{\epsilon}_{x}, \widetilde{\epsilon}_{y}, \widetilde{\gamma}_{x y}\right)$ of the smoothed strain tensor at the generic node $j . \widetilde{\boldsymbol{\epsilon}}_{j}$ can be computed as

$$
\widetilde{\boldsymbol{\epsilon}}_{j}=\left[\begin{array}{c}
\widetilde{\epsilon}_{y} \\
\widetilde{\epsilon}_{y} \\
\widetilde{\gamma}_{x y}
\end{array}\right]=\left[\begin{array}{c}
\frac{\partial D_{x}}{\partial x} \\
\frac{\partial D_{y}}{\partial y} \\
\frac{\partial D_{x}}{\partial y}+\frac{\partial D_{y}}{\partial x}
\end{array}\right]
$$

Under the hypothesis of plane stress and linear elastic material with young modulus $E$ and poisson coefficient $\nu$, the vector of the three components $\left(\widetilde{\sigma}_{x}, \widetilde{\sigma}_{y}, \widetilde{\tau}_{x y}\right)$ of the smoothed stress tensor $\tilde{\boldsymbol{\sigma}}_{j}$ reads

$$
\widetilde{\boldsymbol{\sigma}}_{j}=\left[\begin{array}{c}
\widetilde{\sigma}_{x} \\
\widetilde{\sigma}_{y} \\
\widetilde{\tau}_{x y}
\end{array}\right]=\frac{E}{1-\nu^{2}}\left[\begin{array}{ccc}
1 & \nu & 0 \\
\nu & 1 & 0 \\
0 & 0 & \frac{1-\nu}{2}
\end{array}\right]\left[\begin{array}{c}
\widetilde{\epsilon}_{y} \\
\widetilde{\epsilon}_{y} \\
\widetilde{\gamma}_{x y}
\end{array}\right]
$$

By similar formulae it is possible to compute the smoothed stress tensor components for $3 \mathrm{D}$ or $2 \mathrm{D}$ plain strain linear models. 
This procedure is very simple for linear elastic materials and could be easily extended to compute the stress of non linear elastic materials (such as hyperelastic materials). In case of materials where the stress is function of the deformation history of the component (for instance plasticity) or of other quantities (for instance deformation velocity), this approach can be quite complex if applicable at all.

\section{Stress gradient recovery methods}

In this section four different techniques for stress recovery are described. In some cases more than one implementation can be found in the literature for the same basic approach. In these cases, the different approach are briefly described and the approach followed in this paper is indicated. In one case (double $\mathrm{ZZ}$ recovery, Sect. 3.2), a novel implementation is presented.

\subsection{Shape functions based recovery}

The shape functions based recovery $(S F B R)$ is employed in some commercial software [23] and consists in deriving the smoothed value of stress at nodes by using the derivative of the shape functions.

In the finite element method, the value of a function $f$ (displacement, temperature, ...) can be computed at any point of one element starting from the values $f_{j}$ of the function at nodes as

$f=\sum_{j} N_{j}(x, y, z) f_{j}$, with $\left\{\begin{array}{l}x=\sum_{j} N_{j}(r, s, t) x_{j} \\ y=\sum_{j} N_{j}(r, s, t) y_{j} \\ z=\sum_{j} N_{j}(r, s, t) z_{j}\end{array}\right.$

where the expressions on the right represent the isoparametric transformation with respect to the standard variables $r, s$ and $t$. By defining the Jacobian matrix of the isoparametric transformation $\boldsymbol{J}$ as

$$
\boldsymbol{J}=\left[\begin{array}{l}
\sum_{j} \frac{\partial N_{j}}{\partial r} x_{j} \sum_{j} \frac{\partial N_{j}}{\partial r} y_{j} \sum_{j} \frac{\partial N_{j}}{\partial r} z_{j} \\
\sum_{j} \frac{\partial N_{j}}{\partial s} x_{j} \sum_{j} \frac{\partial N_{j}}{\partial s} y_{j} \sum_{j} \frac{\partial N_{j}}{\partial s} z_{j} \\
\sum_{j} \frac{\partial N_{j}}{\partial t} x_{j} \sum_{j} \frac{\partial N_{j}}{\partial t} y_{j} \sum_{j} \frac{\partial N_{j}}{\partial t} z_{j}
\end{array}\right]
$$

the gradient of $f$ reads

$$
\boldsymbol{\nabla} \boldsymbol{f}=\boldsymbol{J}^{-1}\left[\begin{array}{l}
\sum_{j} \frac{\partial N_{j}}{\partial r} f_{j} \\
\sum_{j} \frac{\partial N_{j}}{\partial s} f_{j} \\
\sum_{j} \frac{\partial N_{j}}{\partial t} f_{j}
\end{array}\right]
$$

By replacing $f_{j}$ with the components of the smoothed stress at nodes in eq. 9, the stress gradient at any node can be computed. For each node a different value of the stress gradient is computed from each of the elements connected to that node. The gradient is then averaged by applying eq. 1 where instead of the stress the stress gradient is considered. In most cases, the nodal averaged stress value (eq. 1) is utilized in eq. 9 as smoothed stress value to replace $f_{j}$.

\subsection{Double ZZ patch recovery}

The idea is to apply the ZZ patch recovery (Sect. 2.2) twice. Different implementations of the double $\mathrm{ZZ}$ patch recovery $(D Z Z P R)$ can be found.

In [43] the ZZ patch recovery is applied after each derivation. The value of stress smoothed over a patch is derived with respect to the spatial coordinates. A constant (for linear elements) or linear varying (for quadratic elements) stress gradient over the patch is obtained. These values of the stress gradient are then interpolated over larger patches constructed over two levels of elements surrounding the vertex node.

This approach has two major shortcomings. First the search of the elements of the patches and the treatment of vertex and non-vertex nodes is rather complicated and leads to relative large matrices to be inverted for each patch. Second, by employing larger patches, the extrapolated values of the stress gradients at boundary nodes (nodes lying on $\partial \Omega$ ) have lower accuracy as shown in [43].

In [33], the interpolating function of the stresses (gradient) is derived at the location where the stress gradient (Hessian) has to be computed. In this case, just one level of surrounding elements can be considered, but in case of linear elements and linear interpolating functions, the stress gradient is constant over the patch.

A different implementation is presented in [11], where structured patches of the same shape of the parent elements are used. Then, the shape functions of the (quadratic) parent elements are mapped on the patch and derived in order to obtain the Hessian. In this way, the use of $C^{1}$ elements is avoided, while sufficient continuous strain fields are obtained. This approach, however, requires large patches with stringent requirements both on patches shapes and orientation.

In [13], the ZZ patch recovery is applied to the recovered values of stresses (gradient) at integration points. This method can use the same patch for stress and stress gradient. However, in [13] numerical proofs have been given that this method is less effective than the method described in the following section (Sect. 3.3) 
In this paper, to use the same patches of just one level of surrounding elements both for stress and stress gradient recovery and to take advantage of the good properties of the Gauss points of the underlying mesh, a different implementation is considered.

The smoothed value of stress obtained by the $Z Z$ patch recovery (Sect. 2.2) is derived for the Gauss points of each element by applying eq. 9 . In this way, a good estimation of the stress gradient should be obtained at the location of the Gauss point where the gradients are usually well approximated. The values of the stress gradient at nodes are then computed by applying the $Z Z$ patch recovery as shown in Sec. 2.2 where instead of the stress the stress gradient is considered.

It can be observed that the same matrices $\boldsymbol{P}$ can be used and the pseudo-inverse operation can be performed just once to interpolate stress and stress gradient. The stress gradient is function of two levels of patches as the stress values have to be reconstructed at each node of the patches before the reconstruction of the stress gradient can be performed.

\subsection{Double polynomial preserving recovery}

This method consists in computing the second derivative of the smoothing functions used in the polynomial preserving recovery applied twice. Different implementation of the double polynomial preserving recovery $(D P P R)$ can be found.

In [43] the Hessian is recovered by computing the second derivatives of the interpolating functions of the displacements (Sect. 2.3). To obtain the stress gradient, the derivative of eq. 6 has to be computed. By considering also eq. 5 , the smoothed stress gradient for a plane stress problem reads

$$
\begin{aligned}
\widetilde{\boldsymbol{\nabla}}_{j}= & {\left[\begin{array}{cc}
\frac{\widetilde{\partial \sigma}_{x}}{\partial x} & \frac{\widetilde{\partial \sigma_{x}}}{\partial y} \\
\frac{\widetilde{\partial \sigma_{y}}}{\partial x} & \frac{\widetilde{\partial \sigma_{y}}}{\partial y} \\
\frac{\partial \tau_{x} y}{\partial x} & \frac{\widetilde{\partial \tau_{x} y}}{\partial y}
\end{array}\right]=\frac{E}{1-\nu^{2}}\left[\begin{array}{ccc}
1 & \nu & 0 \\
\nu & 1 & 0 \\
0 & 0 & \frac{1-\nu}{2}
\end{array}\right] } \\
& {\left[\begin{array}{cc}
\frac{\partial^{2} D_{x}}{\partial x^{2}} & \frac{\partial^{2} D_{x}}{\partial x \partial y} \\
\frac{\partial^{2} D_{y}}{\partial x \partial y} & \frac{\partial^{2} D_{y}}{\partial y^{2}} \\
\frac{\partial^{2} D_{x}}{\partial x \partial y}+\frac{\partial^{2} D_{y}}{\partial x^{2}} & \frac{\partial^{2} D_{x}}{\partial y^{2}}+\frac{\partial^{2} D_{y}}{\partial x \partial y}
\end{array}\right] }
\end{aligned}
$$

In [13], the Hessian is recovered by applying the polynomial preserving recovery to the recovered values of gradient at nodes (Sect. 2.3). If the stress gradient has to be obtained, the polynomial preserving recovery can be applied to the recovered values of stresses (eq. $6)$.

In this paper, the last version is implemented.

\subsection{Stress field gradient analysis}

The stress field gradient analysis technique ( $S F G A$ ) has been presented in [49] as a procedure to have a simple and accurate estimation of the stress gradient also in the preliminary analysis with low order finite elements. The method is conceived to be a post processing addon for commercial finite element codes. Starting from the averaged nodal stress (Sect. 2.1), the stress gradient is computed from the locations and stress values of a central node and a number of surrounding nodes.

Let us indicate with $\boldsymbol{x}_{j}$ the location of a node, $\boldsymbol{x}_{l}$ the location of a surrounding node,

$d_{l j}=\sqrt{\left(\boldsymbol{x}_{l}-\boldsymbol{x}_{j}\right)^{T}\left(\boldsymbol{x}_{l}-\boldsymbol{x}_{j}\right)}$ the distance between the central node and the surrounding node and $\boldsymbol{n}_{l j}=$ $\left(\boldsymbol{x}_{l}-\boldsymbol{x}_{j}\right) / d_{l j}$ the corresponding versor. Then, the directional derivative of stress in direction $\boldsymbol{n}_{l j}$ reads [49]

$$
\frac{\partial \widetilde{\sigma_{j}}}{\partial \boldsymbol{n}_{l j}}=\lim _{\boldsymbol{x}_{l} \rightarrow \boldsymbol{x}_{j}} \frac{\widetilde{\sigma}_{l}-\widetilde{\sigma}_{j}}{d_{l j}} \simeq \frac{\left(\boldsymbol{x}_{l}-\boldsymbol{x}_{j}\right)^{T}}{d_{l j}}\left[\begin{array}{c}
\frac{\widetilde{\partial \sigma}_{j}}{\partial x} \\
\frac{\widetilde{\partial \sigma}_{j}}{\partial y} \\
\frac{\partial \sigma_{j}}{\partial z}
\end{array}\right]=\boldsymbol{n}_{l j}^{T} \widetilde{\boldsymbol{\nabla} \sigma_{j}}
$$

One equation of the kind of eq. 11 can be written for any node surrounding node $j$ and a overdetermined system of equations can be written as

$$
\boldsymbol{A} \nabla \sigma_{j}=\boldsymbol{b}
$$

with

$$
\boldsymbol{A}=\left[\begin{array}{c}
\boldsymbol{n}_{l_{1} j} \\
\boldsymbol{n}_{l_{2} j} \\
\vdots \\
\boldsymbol{n}_{l_{n} j}
\end{array}\right] \quad \boldsymbol{b}=\left[\begin{array}{c}
\boldsymbol{x}_{l_{1}}-\boldsymbol{x}_{j} \\
\boldsymbol{x}_{l_{2}}-\boldsymbol{x}_{j} \\
\vdots \\
\boldsymbol{x}_{l_{n}}-\boldsymbol{x}_{j}
\end{array}\right]
$$

where $l_{1}, l_{2}, \ldots, l_{n}$ are the $n$ surrounding nodes of node $j$. From eq. 11, the gradient of each stress component at node $j$ can be computed as [3] 
$\nabla \sigma_{j}=\boldsymbol{A}^{\dagger} \boldsymbol{b}$

Where $\boldsymbol{A}^{\dagger}$ represents the pseudoinverse of matrix $\boldsymbol{A}$. The number $n$ of considered surrounding nodes must comply with $n \geq 2$ for $2 \mathrm{D}$ models and $n \geq 3$ for $3 \mathrm{D}$ models. The sampling nodes are chosen by considering a circle (or sphere) of radius $\rho$ around the considered node. If the number of nodes at a distance less than $\rho$ is less than a minimum number of nodes, $\rho$ is incremented to include a sufficient number of nodes. A minimum number of 5 or 9 nodes for respectively $2 \mathrm{D}$ and $3 \mathrm{D}$ elements is considered.

\section{Radial basis function networks}

Radial basis function networks (RBFN) can approximate any regular function [16] and the training is faster than that of a multilayer perceptron neural network [28]. The design of an RBFN is a curve-fitting (approximation) problem in a high-dimensional space; the approach is similar to the use of a multidimensional surface to interpolate data. A conceptual scheme of the Radial Basis Function network is reported in Fig. 1.

Radial Basis Function networks can be used for the approximation of a function and its derivatives (scattered data interpolation) $[18,36]$. Different basis functions known as Gaussian, Multiquadrics and Inverse Multiquadrics are generally used [28]. In problems related to structural analysis, also Cubic, Thin plate splines and Wendland functions are used [10, 26]. These basis functions are continuously differentiable and integrable, this is useful for the approximation of the derivatives. The Multiquadrics basis function approach yields a superior accuracy in the approximation of the derivatives as shown in [8].

The original RBFN method requires that there must be as many RBFN centres as the training data points, but a reduced set of centers can be successfully used.

In the case of a scalar output the model can be expressed ( $n$ is 2 for $2 \mathrm{D}$ problems, and 3 for $3 \mathrm{D}$ problems) by

$\hat{f}(\boldsymbol{x}, \boldsymbol{w})=\sum_{i=1}^{r} h_{i}(\boldsymbol{x}) w_{i}$

The most widely used basis function $h_{i}$ are respectively the Gaussian basis functions (Fig. 2)

$\rho_{i}=\sum_{j=1}^{n}\left(x_{j}-c_{i j}\right)^{2} \quad h_{i}(\boldsymbol{x})=\exp \left(-\left(\rho_{i} \sigma\right)^{2}\right)$

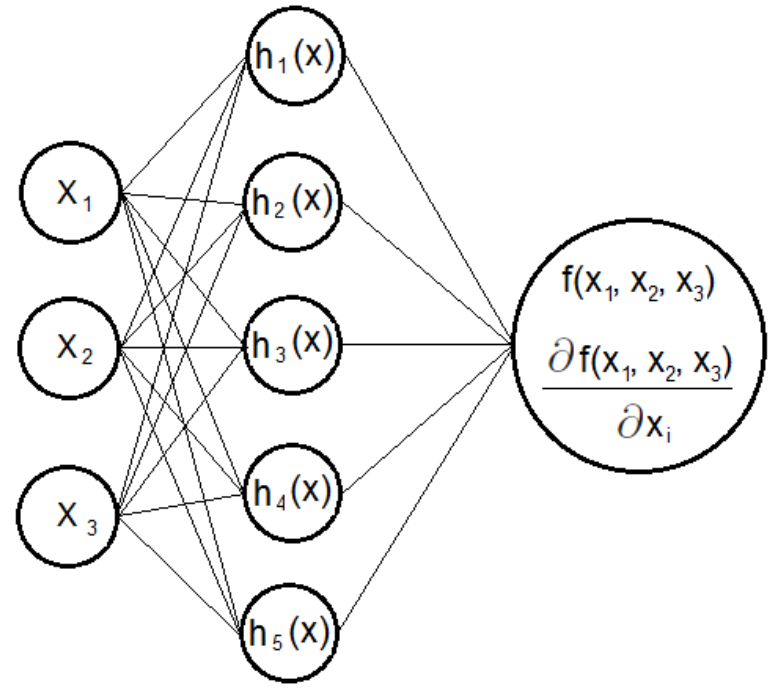

Fig. 1 A Radial Basis Function Network for multiple inputs (3D) and one output with derivatives.
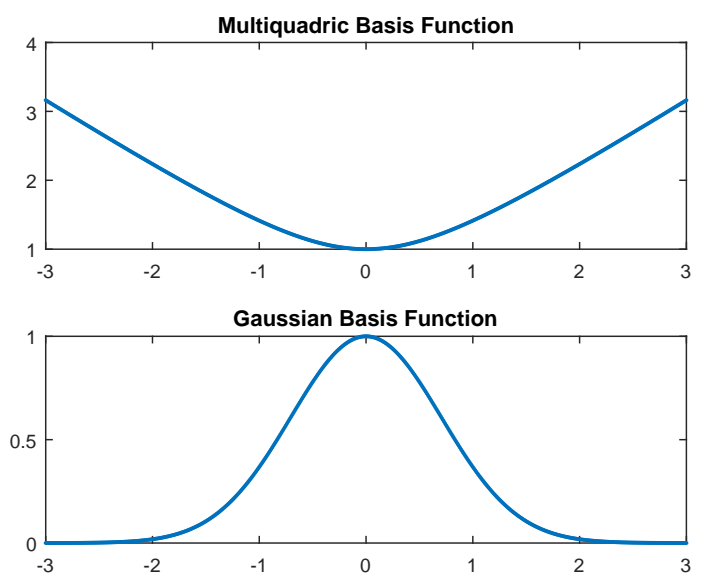

Fig. 2 The most frequently used basis functions for RBFN.

and the Multiquadric function [6] (Fig. 2)

$\rho_{i}=\sum_{j=1}^{n}\left(x_{j}-c_{i j}\right)^{2} \quad h_{i}(\boldsymbol{x})=\left(1+\left(\rho_{i} \sigma\right)^{2}\right)^{1 / 2}$

Being the most used and particularly suitable for derivative approximation, these two basis functions will be considered in the remainder of the paper.

Every basis function $h_{i}(\boldsymbol{x}), i=1, \ldots, r$ depends only on the scaled distance between the input $\boldsymbol{x}$ and a centre $\boldsymbol{c}_{i}$ scaled by the shape parameter $\sigma$ that has to be properly selected [47]. There is experimental evidence showing that the accuracy of the approximation strongly depends on the value of the shape parameter $\sigma$. If $\sigma$ is too small the condition number of the resulting linear system increases giving rise to numerical instabilities and 
loss of precision. By decreasing the shape parameter $\sigma$, the RMS (root mean square) error of the fit drops to a minimum and then grows thereafter, i.e. there exists an optimal shape parameter that will yield minimum RMS error for the fitted function [45].

As pointed out in [6], the choice of centres which determines the number of free parameters is of crucial importance. Too few centres in the network may be not capable of generating a good approximation to the target function, on the other hand, too many centres may fit misleading variations due to noise in the data. This is the bias/variance dilemma typical of this type of problems. In the present application, a fixed number $(r=1000)$ of centers has been used. The centers have been selected by using a Sobol low discrepancy sequence [27] in order to assure a uniform distribution in the two/three dimensional space.

\section{Examples and results}

Two examples, one in two and one in three dimensions, are considered to asses the performances of the described recovery techniques for stress and stress gradient. In many papers [12], analytical functions solved over different meshes are used to isolate the error due to the recovery procedure from the numerical errors of the finite elements model. In the examples reported in this section, the finite element solution is considered in order to evaluate the actual level of approximation that can be obtained in an engineering problem. In particular, if the error is always decreasing with mesh refinement, it means that the finite element solution has a small numerical error compared with the recovery error. If the error is not decreasing when the mesh dimension is below a certain value, it means that the numerical error of the finite element solution is greater than the recovery error.

Table 1 reports the elements used to solve the two examples. For quad and brick elements, the reduced integration has been considered. In this case, for regular meshes, the Gauss points coincide with the superconvergent points and for irregular meshes, the Gauss points can be still considered optimal sampling points [57].

Each of the two examples can be solved analytically. Thus, the accuracy of the reconstructed fields can be compared with the analytical solution. The finite element models are solved for the considered elements for five different element sizes. The reconstruction error is evaluated both in the interior of the domain and on the boundary where stresses and stress gradients assume the maximum values.

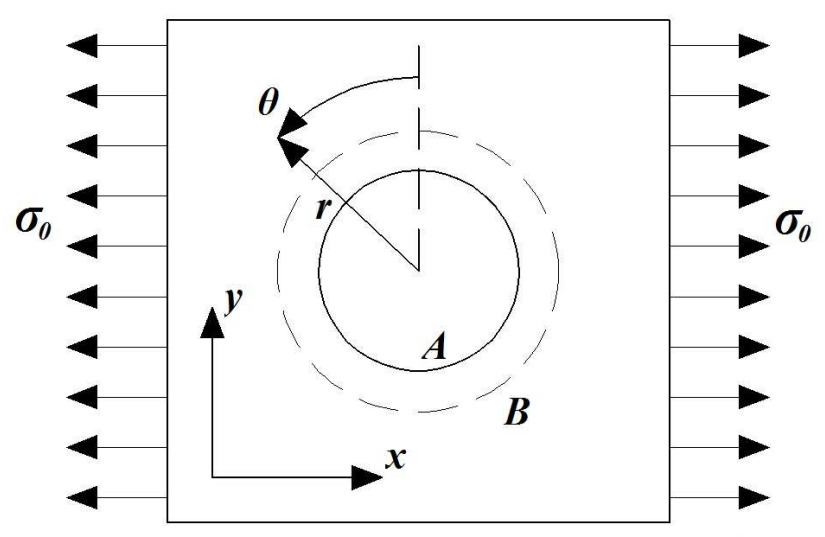

Fig. 3 Infinite plate with hole under uniaxial stress. Data: $\sigma_{0}=100 \mathrm{MPa}$, hole radius $=5 \mathrm{~mm}$, plate dimensions in the finite element model 1000x1000 mm. A: hole boundary. B: circumference at $2 \mathrm{~mm}$ from the hole boundary.

The considered error measure evaluates the mean error along two node lines (lines shapes and locations are defined for each example). One line is located inside the domain, the other one on the boundary. For each line the mean error is defined as

$\bar{e}=\frac{\sum_{j=1}^{n}\left\|\widetilde{\boldsymbol{g}}_{j}-\boldsymbol{g}_{j}\right\|}{\sum_{1}^{n}\left\|\boldsymbol{g}_{j}\right\|}$

where $n$ is the number of nodes on the line and $\boldsymbol{g}$ is the considered field $(\boldsymbol{g}=\boldsymbol{\sigma}$ for the vector of the stress component, $\boldsymbol{g}=\boldsymbol{\partial} \boldsymbol{\sigma} \backslash \boldsymbol{\partial x}, \boldsymbol{g}=\boldsymbol{\partial} \boldsymbol{\sigma} \backslash \boldsymbol{\partial} \boldsymbol{y}$ and $\boldsymbol{g}=\boldsymbol{\partial} \boldsymbol{\sigma} \backslash \boldsymbol{\partial z}$ for the vectors of the stress gradients with respect to $x$, $y$ and $z$ respectively). Eq. 18 represents a normalized error with respect to the average value of the function on the line and does not depend on the number of considered nodes.

$5.12 \mathrm{D}$ example: infinite plate with hole under uniaxial stress

The first example is the well known analysis of a infinite plate with hole under uniaxial stress. The problem is described in Fig. 3. The analytical solution of the stress field in polar coordinates reads

$$
\begin{aligned}
\sigma_{r} & =\frac{1}{2} \sigma_{0}\left(\cos (2 \theta)\left(\frac{3 a^{4}}{r^{4}}-\frac{4 a^{2}}{r^{2}}+1\right)-\left(\frac{a^{2}}{r^{2}}+1\right)\right) \\
\sigma_{\theta} & =-\frac{1}{2} \sigma_{0}\left(\cos (2 \theta)\left(\frac{3 a^{4}}{r^{4}}+1\right)+\left(\frac{a^{2}}{r^{2}}+1\right)\right) \\
\tau_{r \theta} & =-\frac{1}{2} \sigma_{0} \sin (2 \theta)\left(-\frac{3 a^{4}}{r^{4}}+\frac{2 a^{2}}{r^{2}}+1\right)
\end{aligned}
$$


Table 1 Element types considered in the analysis

\begin{tabular}{ccccccc}
\hline $\begin{array}{c}\text { Element } \\
\text { type }\end{array}$ & Dimension & $\begin{array}{c}\text { Number } \\
\text { of nodes }\end{array}$ & $\begin{array}{c}\text { Number of } \\
\text { integration } \\
\text { points }\end{array}$ & Type & Shape & ABAQUS element type \\
\hline T3 & 2D & 3 & 1 & linear & triangle & CPS3 \\
Q4 & 2D & 4 & 1 & linear & quad & CPS4R \\
T6 & 2D & 6 & 2 & quadratic & triangle & CPS6 \\
Q8 & 2D & 8 & 4 & quadratic & quad & CPS8R \\
T4 & 3D & 4 & 1 & linear & tetra & C3D4 \\
B8 & 3D & 8 & 1 & linear & brick & C3D8R \\
T10 & 3D & 10 & 4 & quadratic & tetra & C3D10 \\
B20 & 3D & 20 & 8 & quadratic & brick & C3D20R \\
\hline
\end{tabular}

where $\sigma_{0}$ is the uniaxial stress value far from the hole and $a$ is the radius of the hole. The solution in polar coordinates of eq. 19 has been rotated to be expressed in the $x-y$ reference system of Fig. 3 as

$$
\left[\begin{array}{cc}
\sigma_{x} & \tau_{x y} \\
\tau_{x y} & \sigma_{y}
\end{array}\right]=\boldsymbol{R}\left[\begin{array}{cc}
\sigma_{r} & \tau_{r \theta} \\
\tau_{r \theta} & \sigma_{\theta}
\end{array}\right] \boldsymbol{R}^{T}, \quad \boldsymbol{R}=\left[\begin{array}{cc}
\cos (\theta) & -\sin (\theta) \\
\sin (\theta) & \cos (\theta)
\end{array}\right]
$$

being $\boldsymbol{R}$ the rotation matrix. The analytical expression of the stress gradient in the $x-y$ reference system can be obtained by deriving eq. 20. This derivation can be performed by a symbolic solver, the expression is quite complicated and is not reported for sake of space.

In Fig. 3, the two circumferences labelled $A$ and $B$ represent the two locations where the error is evaluated ( $A$ is on the boundary of the hole, $B$ is inside the domain at $2 \mathrm{~mm}$ from the hole).

The example is solved by using ABAQUS Standard ver. 6.14 with full precision nodal output. The mesh pattern used for the analysis for triangular and quad elements is shown in Fig. 4. The triangular mesh pattern has been obtained by dividing the quad elements along one diagonal. In this way, the number of nodes (i.e. degrees of freedom) in the zone of interest is the same for the two kinds of mesh for linear elements and the quadratic triangles have one more node for each quadrangular cell than the eight nodes quad. By this choice, for the same number of nodes on the hole boundary, the computational cost of the solution is almost the same for all the considered meshes. The example is solved considering 32, 64, 128, 256 and 512 nodes on the hole boundary, corresponding to an indicative mesh size of 1 , $0.5,0.25,0.125$ and $0.0625 \mathrm{~mm}$ for linear elements and $2,1,0.5,0.25$ and $0.125 \mathrm{~mm}$ for quadratic elements. The meshes are refined maintaining the same kind of patterns.

The radial basis neural network used for this example has been constructed, for any mesh size and type of elements, by considering 10 circles of nodes as training

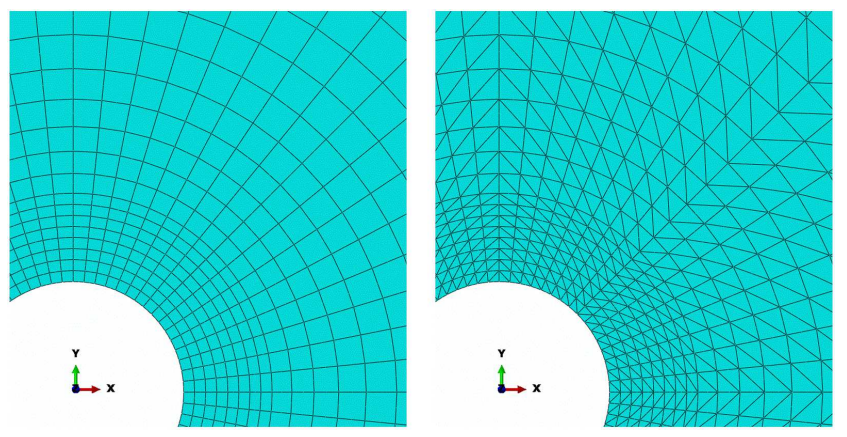

Fig. 4 Detail of the mesh patterns around the hole used for the solution of the infinite plate with hole example. The figure reports the meshes with element size of $0.5 \mathrm{~mm}$, corresponding to 64 and 128 nodes on the hole border for linear and quadratic elements respectively

points. To improve generalization, the number of centres has been limited to the minimum between the $60 \%$ of the training points and 1000. The centres have been evenly distributed in the region defined by the training points. The activation functions are Gaussians to reconstruct quantities inside the domain and multiquadric for the reconstruction on the boundary. The shape parameter $\sigma$ has been optimized in order to minimize the SSD error on the training set. The optimization proess has been repetated for any mesh size, type of element and circle of nodes. The centres have been positioned according to a quasi-uniform sobol sequence [27].

In Fig. 5, 6, 7 and 8 the results of the analysis are reported in bi-logarithmic scale for the four considered meshes. For stress gradients, for sake of space only errors on the reconstruction of $\partial \boldsymbol{\sigma} \backslash \partial y$ are reported. Errors on the reconstruction of $\partial \boldsymbol{\sigma} \backslash \partial x$ are very similar.

Quadratic meshes, as expected, show better results than the corresponding linear meshes for any reconstruction technique both with respect to stresses and stress gradients. Quad elements perform slightly better than the corresponding triangular elements.

Referring to stress reconstruction, in the region inside the domain (path B of fig. 3) all of the reconstruction techniques show good performances, with the 

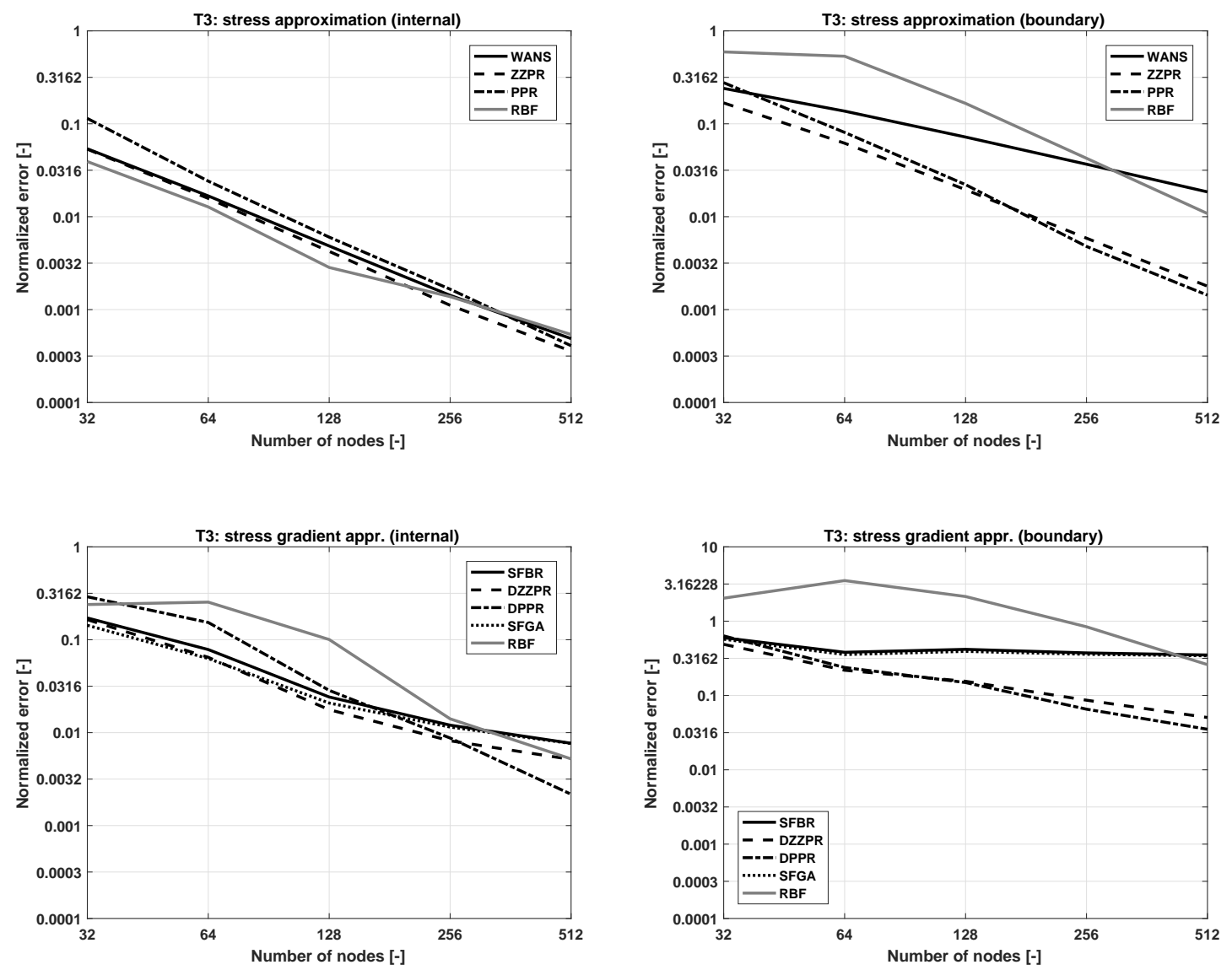

Fig. 5 T3 elements: normalized mean error (eq. 18) as function of the number of nodes on the considered line. Top: stress estimation (Left: path B of fig. 3, Right path A of fig. 3). Bottom: stress gradient estimation (Left: path B of fig. 3, Right path A of fig. 3).

nodal average that has slightly worse results for T3 and T6 elements. On the boundary region (path A of fig. 3 ), the nodal average has a clearly lower rate of convergence of the other techniques. In all considered situations, $\mathrm{ZZ}$ patch recovery and polynomial preserving recovery show similar performances, with the $\mathrm{ZZ}$ patch recovery that has smaller errors with quad elements. The radial basis function network has generally good performances, especially if quadrilateral elements are considered. In this case, RBF shows the best performances.

The reconstruction of the stress gradient shows, as expected, higher values of error with respect to the reconstruction of the stress. The shape function based recovery and the stress field analysis show the worst performances and are not able to reconstruct the stress gradient on the boundary when T3 and Q4 elements are employed. The modified version of the double $\mathrm{ZZ}$ patch recovery and the double polynomial preserving recovery are the best performing with very close errors for triangular elements and better results for the double ZZ patch recovery for quad elements. Again, the radial basis function network shows generally good performances especially if quad elements are considered. In case of triangular linear element, RBF seems not able to get the recostruction of the stress gradient. Conversely, very good performances are obtained for bilinear quad elements.

Both for stress and stress gradient recovery, the error seems to be bounded by a value around 0.0003 . This limit is due to the approximation of the finite element model in which the plate is large with respect to the hole but not infinite. As a test, the analytical solution at grid points has been used for the extrapolation and smaller errors have been found (the results are not reported for sake of space). This means that the best 

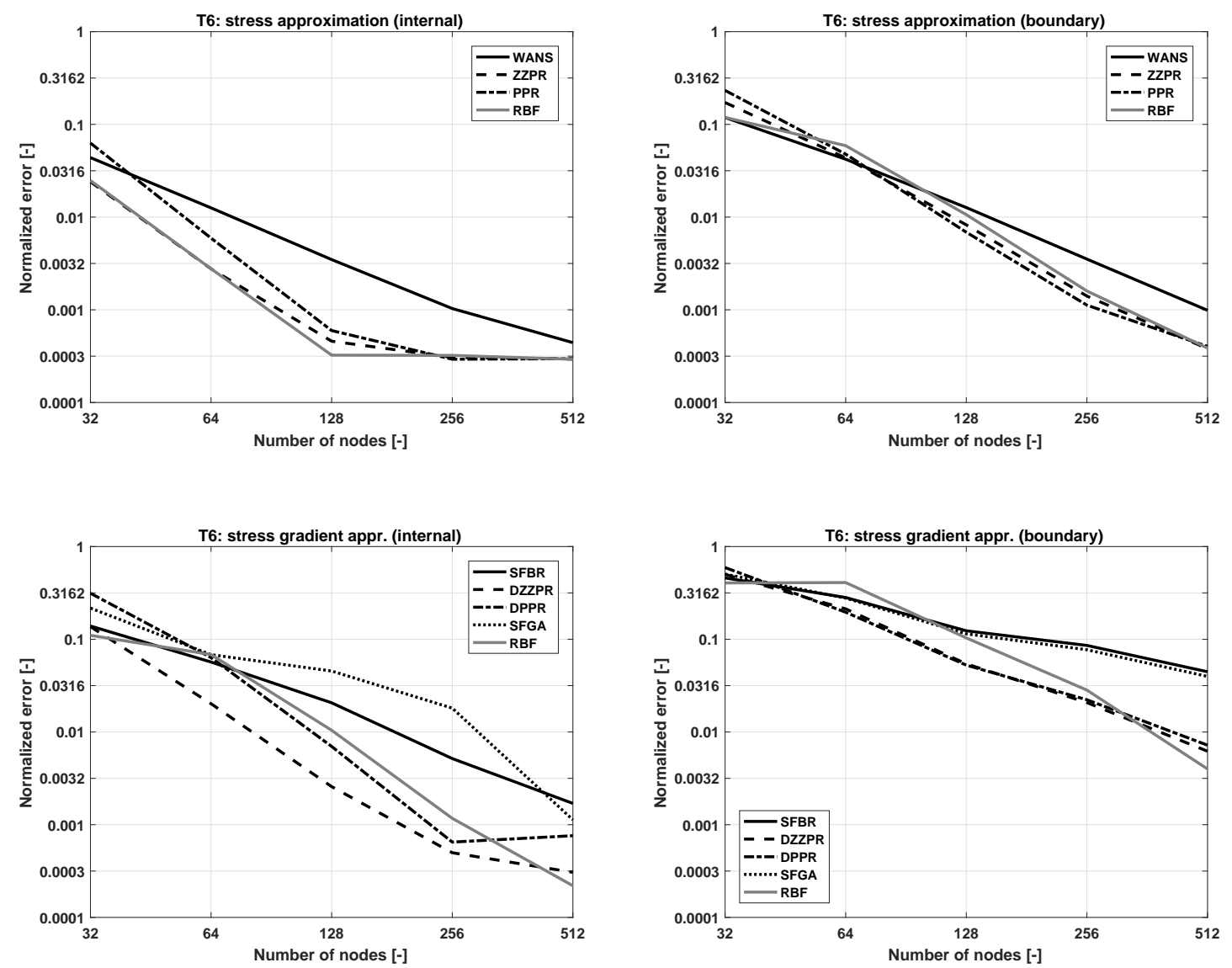

Fig. 6 T6 elements: normalized mean error (eq. 18) as function of the number of nodes on the considered line. Top: stress estimation (Left: path B of fig. 3, Right path A of fig. 3). Bottom: stress gradient estimation (Left: path B of fig. 3, Right path A of fig. 3).

performing techniques are able to exploit the accuracy of the underlying finite element solution.

In table 2 the rates of convergence of the reconstruction techniques are reported. These rates have been computed by linearly interpolating the error trends reported in Fig. 5, 6, 7 and 8 in the ranges where the bound value of the error is not reached. As expected, ZZPR and PPR show superconvergent rates of convergence in the stress reconstruction on the internal nodes of the domain for any considered element type. Slightly worse rates of convergence are shown on the border of the domain, but always greater than the rate of convergence of the underlying elements. WANS shows superconvergency only for T3 and Q4 in the stress reconstruction on the internal nodes of the domain. This can be explained by considering that in this particular case this extrapolation technique is a weighted mean of the stress at the unique gauss point of elements connected at the considered node. Referring to stress gradient recovery, DZZPR and DPPR show higher rates of convergence than the underlying elements both inside the domain and on the border. SFBR and SFGA show acceptable rates inside the domain, while very slow rates have been fond on the border. RBF shows convergent rates similar, and sometimes higher, than the best performin techniques both for stress and stress gradient reconstructions.

Fig. 9 shows the reconstructed values of the norm of the vector of the stress components and of the norm of the derivative of the vector of the stress components with respect to $y$ for a $\mathrm{T} 6$ mesh of $0.5 \mathrm{~mm}$ (128 nodes on the circumference of the hole) on the hole boundary. Given the symmetries of the solution, only $90^{\circ}$ have been plotted. The reconstruction has been performed by using the $\mathrm{ZZ}$ patch recovery for stress and the double ZZ patch recovery for stress gradient. The values of 

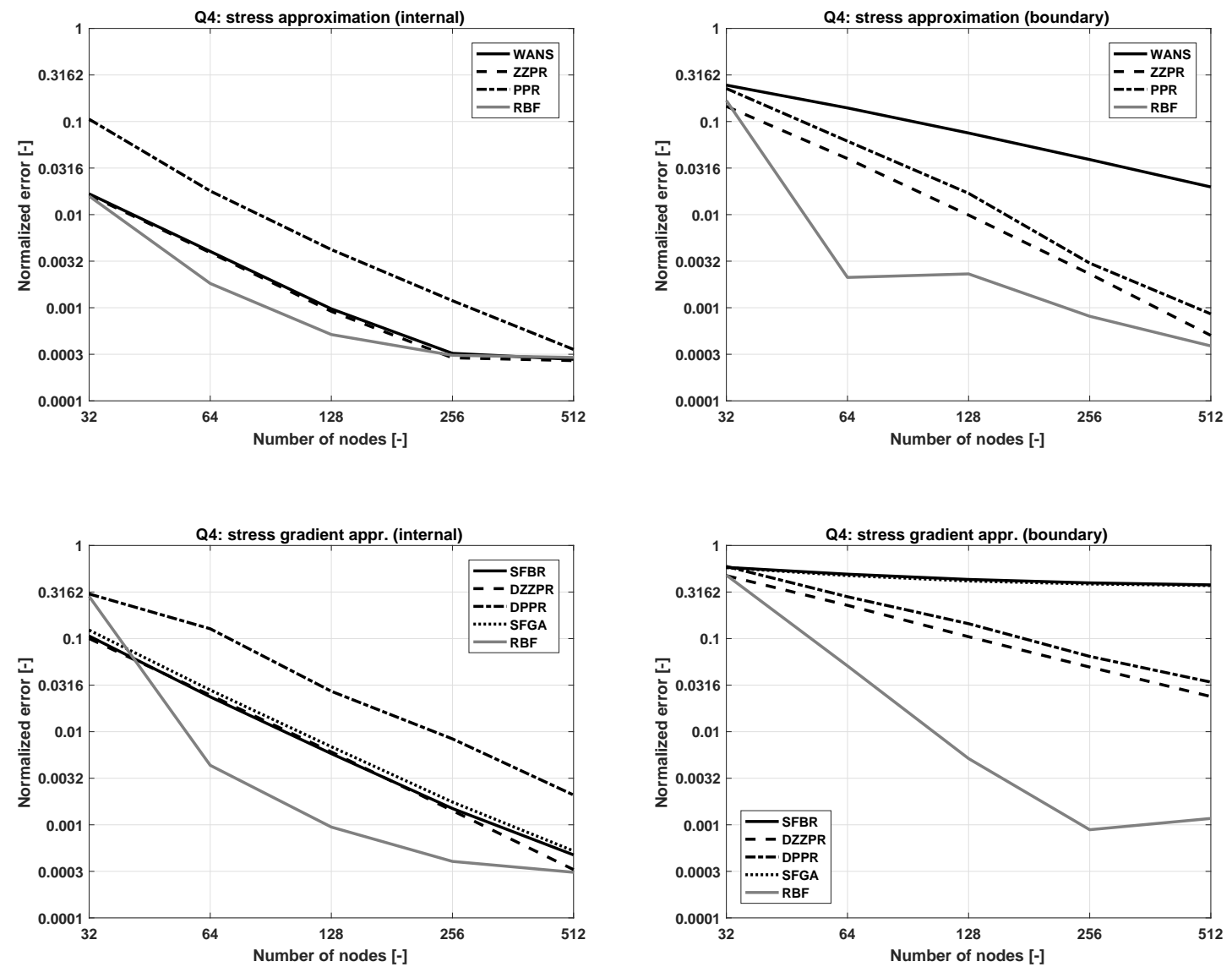

Fig. 7 Q4 elements: normalized mean error (eq. 18) as function of the number of nodes on the considered line. Top: stress estimation (Left: path B of fig. 3, Right path A of fig. 3). Bottom: stress gradient estimation (Left: path B of fig. 3, Right path A of fig. 3).

Table 2 Convergence rates for the considered reconstruction techniques for the infinite plate with hole example. Paths A and B definition in Fig. 3

Internal nodes (path B)

\begin{tabular}{|c|c|c|c|c|c|c|c|c|c|}
\hline & \multicolumn{4}{|c|}{ Stress reconstruction } & \multicolumn{5}{|c|}{ Stress gradient reconstruction } \\
\hline & WANS & ZZPR & PPR & RBF & SFBR & DZZPR & DPPR & SFGA & RBF \\
\hline T3 & 1.71 & 1.82 & 2.01 & 1.56 & 1.17 & 1.30 & 1.85 & 1.09 & 1.52 \\
\hline T6 & 1.83 & 2.86 & 3.36 & 3.13 & 1.61 & 2.70 & 2.97 & 1.71 & 2.80 \\
\hline Q4 & 2.05 & 2.07 & 2.03 & 1.88 & 1.96 & 2.06 & 1.83 & 1.97 & 3.06 \\
\hline Q8 & 2.18 & 3.48 & 3.36 & N.A. & 1.79 & 2.48 & 2.67 & 1.87 & 3.50 \\
\hline
\end{tabular}

Border nodes (path A)

\begin{tabular}{|c|c|c|c|c|c|c|c|c|c|}
\hline & \multicolumn{4}{|c|}{ Stress reconstruction } & \multicolumn{4}{c|}{ Stress gradient reconstruction } \\
\hline & WANS & ZZPR & PPR & RBF & SFBR & DZZPR & DPPR & SFGA & RBF \\
T3 & 0.93 & 1.65 & 1.95 & 1.88 & 0.16 & 0.79 & 1.02 & 0.15 & 1.26 \\
T6 & 1.73 & 2.31 & 2.56 & 2.44 & 0.84 & 1.60 & 1.59 & 0.92 & 2.19 \\
Q4 & 0.91 & 2.04 & 2.04 & 1.89 & 0.16 & 1.08 & 1.04 & 0.16 & 3.05 \\
Q8 & 1.78 & 2.51 & 2.48 & 3.09 & 0.79 & 1.57 & 1.69 & 0.93 & 1.93 \\
\hline
\end{tabular}



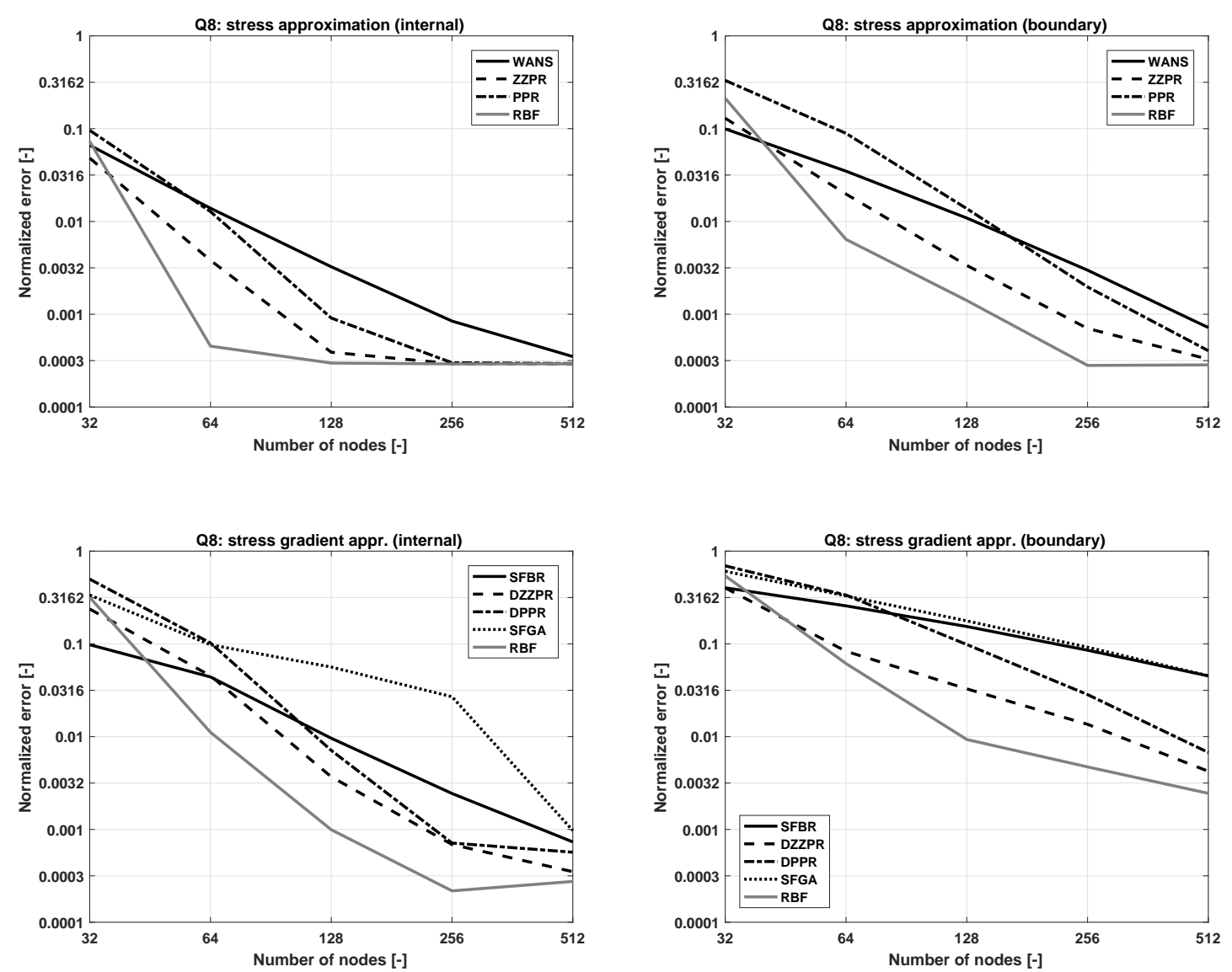

Fig. 8 Q8 elements: normalized mean error (eq. 18) as function of the number of nodes on the considered line. Top: stress estimation (Left: path B of fig. 3, Right path A of fig. 3). Bottom: stress gradient estimation (Left: path B of fig. 3, Right path A of fig. 3).
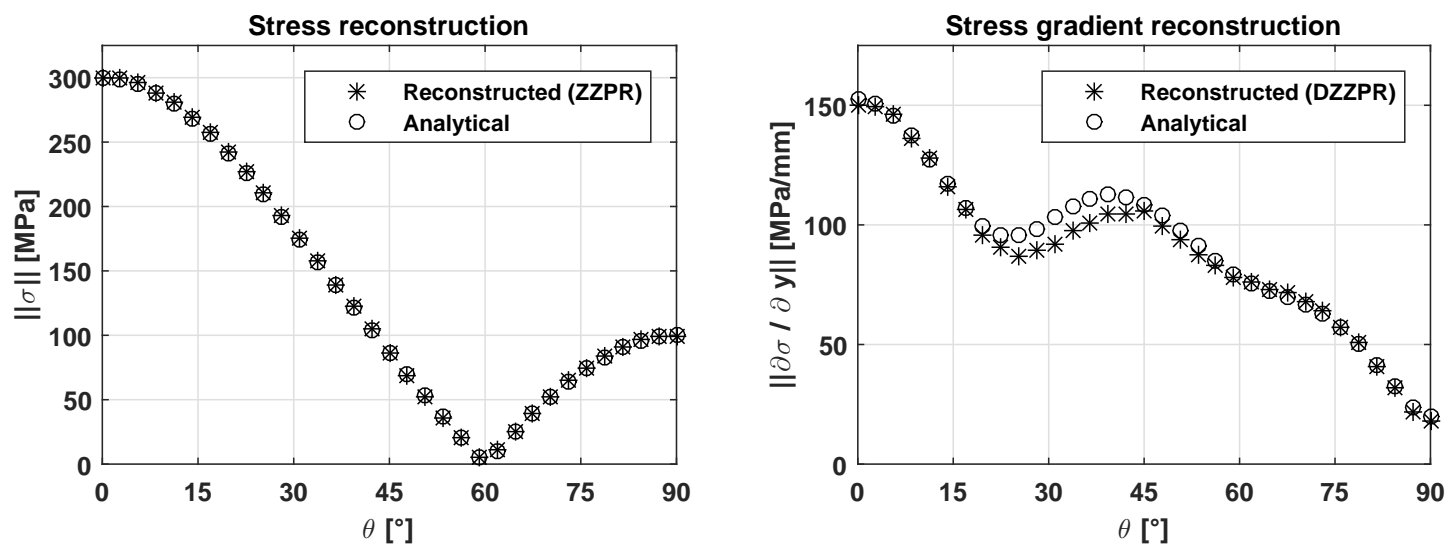

Fig. 9 T6 elements: reconstructed values of stress (left) and stress gradient with respect to $y$ (right) for a mesh size of 0.50 $\mathrm{mm}$ on the boundary of the hole (path A of fig. 3) by using a double ZZ patch recovery. 
the error indices of eq. 18 is 0.010 for the stress and 0.065 for the stress gradient. From the figure, it can be observed that the stress reconstruction is very accurate (error of less than $0.5 \mathrm{MPa}$ at the locatin of the maximum stress of $300 \mathrm{MPa}$ ) and the stress gradient is well reconstructed on the location of its maximum (less than $2.5 \mathrm{Mpa} / \mathrm{mm}$ of difference with a value of 150 $\mathrm{MPa} / \mathrm{mm}$ ) and a maximum error of about $10 \mathrm{MPa} / \mathrm{mm}$.

The meshes required to have a fairly reconstruction of the stress gradient, such as the one used to realized Fig. 9, are impractical for most applications where complex component are usually to be studied with more than one critical region. In practice, well structured meshes are very rare and biased meshes of triangular elements are usually employed. Fig. 10 shows the error as function of the number of nodes on the circumference of the hole for the boundary region when a mesh of T6 elements with a growing factor of 5 times in $10 \mathrm{~mm}$ is used. The computed errors are very close to the errors of Fig. 6, showing a good robustness of the considered methods to reasonable non uniform meshes.

\section{$5.23 \mathrm{D}$ example: rotating thick tube}

This example is a rotating thick tube of infinite length. The problem can be solved analytically under the hypothesis of plane strain. The problem is described in Fig. 11. The analytical solution can be derived in the cylindrical reference frame $r-\theta-z$ with $z$ coincident with the tube axis. The resulting stress is three dimensional with $\tau_{r \theta}=\tau_{r z}=\tau_{\theta z}=0$ and reads

$$
\begin{aligned}
\sigma_{r} & =\frac{(3-2 \nu) \rho \omega^{2} r_{o}^{2}}{8(1-\nu)}\left(1+\frac{1}{r_{i}^{2}}-\frac{r_{i}^{2}}{r^{2}}-\frac{r^{2}}{r_{o}^{2}}\right) \\
\sigma_{\theta} & =\frac{(3-2 \nu) \rho \omega^{2} r_{o}^{2}}{8(1-\nu)}\left(1+\frac{1}{r_{i}^{2}}+\frac{r_{i}^{2}}{r^{2}}-\frac{r^{2}(1+2 \nu)}{(3-2 \nu) r_{o}^{2}}\right) \\
\sigma_{z} & =\frac{\rho \omega^{2} r_{e}^{2}}{4(1-\nu)}\left(1+\frac{1}{r_{i}^{2}}-2 \frac{r^{2}}{r_{o}^{2}}\right)
\end{aligned}
$$

where $r_{i}$ and $r_{o}$ are the inner and outer radius of the tube respectively, $\omega$ is the angular velocity around the axis of the tube and $\nu$ is the Poisson coefficient of the material. The stress tensor in the cylindrical reference system can be rotated in order to obtain the components of the tensor in the $x-y-z$ reference system by applying a rotation similar to eq. 20 . As in the previous example, the stress tensor in the $x-y-z$ reference system can be derived by a symbolic solver and the analytical expressions of the stress gradient can be obtained (not reported for sake of space).
The FE model of the tube is solved by using ABAQUS Standard ver. 6.14 with full precision nodal output. Proper displacement constraints have been considered at the top and bottom surface of the tube to impose a plain deformation of both ends and simulate a infinite length. The mesh patterns used for the analysis are reported in Fig. 12 and are the three dimensional versions of the patterns shown in Fig. 4. The patterns are constants along the axis of the cylinder. The tetrahedral mesh pattern has been obtained by dividing the brick elements as to keep a right angle for each tetrahedron, i.e. four tetrahedrons have been created from each brick. In this way, the number of nodes (i.e. degrees of freedom) in the zone of interest is the same for the two kinds of meshes for linear elements. For quadratic elements, quadratic tetrahedrons have four more nodes for each brick cell than the twenty nodes bricks. The example is solved considering 32, 64, 128, 320 and 640 nodes on the hole boundary at the mid-plane of the cylinder model, corresponding to an indicative mesh size of 1 , $0.5,0.25,0.1$ and $0.05 \mathrm{~mm}$ for linear elements and 2, 1 , $0.5,0.2$ and $0.1 \mathrm{~mm}$ for quadratic elements. The meshes are refined maintaining the same kind of patterns.

The radial basis function network used for this example has been constructed considering as training points the nodes comprised in a distance of 10 or 5 element lengths (respectively for linear and quadratic meshes) from the circles of nodes of lines A and B of Fig. 11. As for the previous example, the number of centres has been chosen equal to the minimum between the $60 \%$ of the training point and 1000. The centres have been evenly distributed in the region defined by the training points accordingly to a quasi-uniform Sobol sequence [27]. The activation functions are Gaussians to reconstruct quantities inside the domain and multiquadric for the reconstruction on the boundary. As in the previous example (Sect. 5.1), the shape paramter has been optimized, for each case, to minimize the SSD error on the training points.

In Fig. 13, 14, 15 and 16 the results of the analysis are reported in bi-logarithmic scale for the four considered meshes. For stress gradients, for sake of space, only errors on the reconstruction of $\partial \boldsymbol{\sigma} \backslash \partial y$ are reported. Given the symmetry of the problem, errors on the reconstruction of $\partial \boldsymbol{\sigma} \backslash \partial x$ have the same values. Errors on the reconstruction of $\partial \boldsymbol{\sigma} \backslash \partial z$ have slightly different behaviours but are always smaller than the errors on the reconstruction of $\partial \boldsymbol{\sigma} \backslash \partial y$.

All the considered reconstruction techniques show large errors in gradient reconstruction when linear tetrahedron are used (Fig. 13) both inside and on the border of the domain. Particularly poor performances are shown by shape function based, stress field analysis 

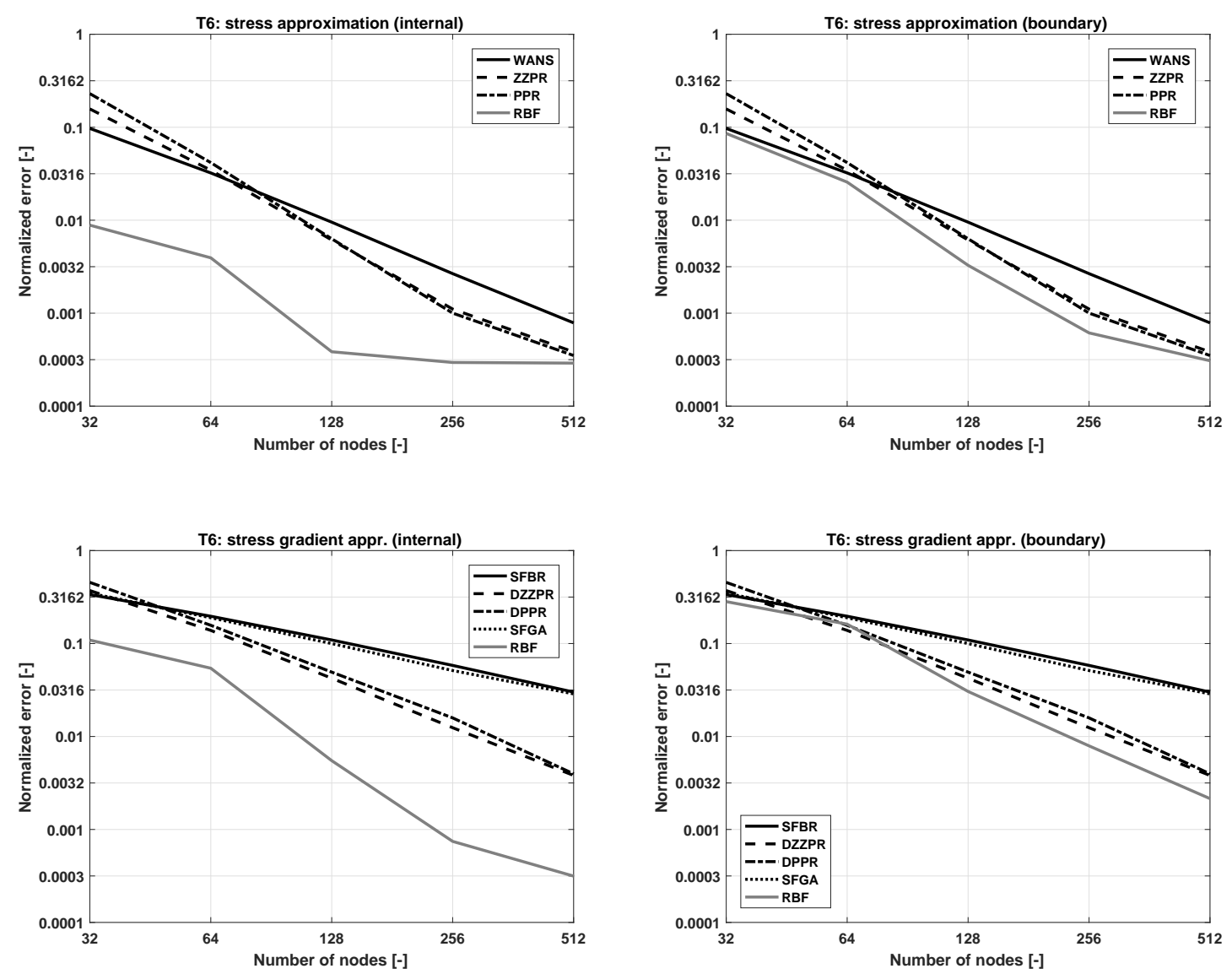

Fig. 10 T6 elements with biased mesh: normalized mean error (eq. 18) as function of the number of nodes on path A of fig. 3. Left: stress estimation. Right: stress gradient estimation.

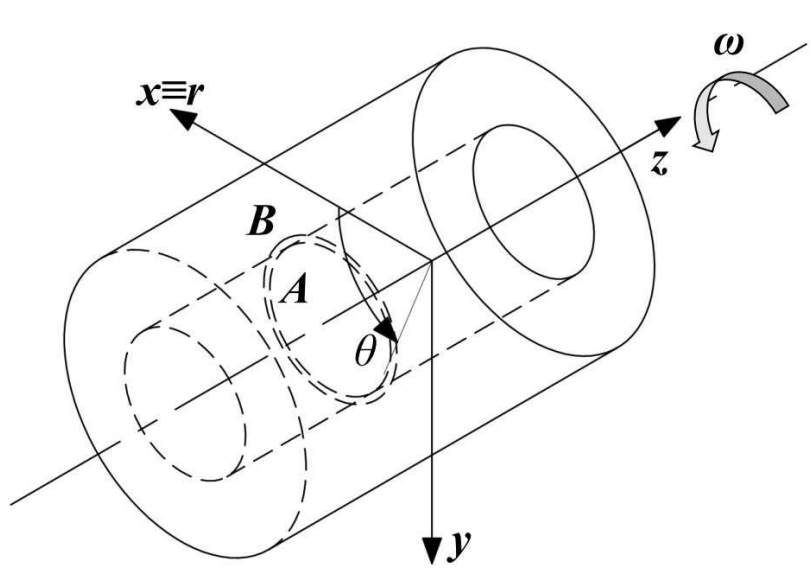

Fig. 11 Rotating thick tube of infinite length. Data: $r_{i}=$ $5 m m, r_{o}=25 m m, \omega=1 s^{-1}, \nu=0.3$. A: circumference on the inner surface of the cylinder. B: circumference at $2 \mathrm{~mm}$ from inner surface of the cylinder.

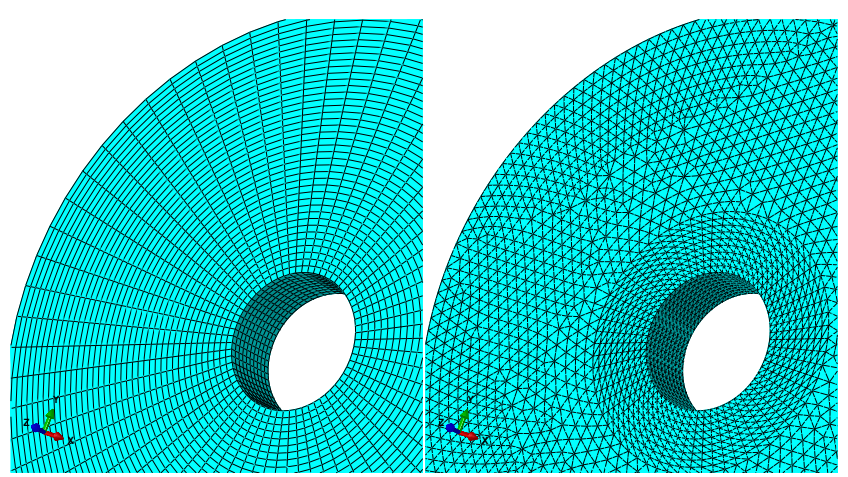

Fig. 12 Detail of the mesh patterns around the hole used for the solution of the rotating thick tube of infinite length. The figure reports the meshes with element size of $0.5 \mathrm{~mm}$. On the top and bottom surfaces of the cylinder, a symmetry constraint is enforced to simulate the infinite length of the tube.

techniques and radial basis function network. With linear brick, better performances are shown by all the tech- 
niques inside the domain. On the border, only double zz patch recovery, radial basis function network and, at least partially, double polynomial preserving techniques show convergence.

Better stress gradient reconstruction is obtained with quadratic elements for all the considered techniques, with the best performances obtained by the double $\mathrm{ZZ}$ patch recovery and the radial basis function network. For this example, no lower bound of the error is reached with mesh refinement, meaning that the FE model is actually describing the analytical problem.

In table 3 the convergence rates of the considered reconstruction techniques are reported. Among the local reconstruction techniques, both in stress and in stress gradient reconstruction, the DZZPR technique shows the higher rates of convergence, which are always greater than the underlying elements. Similar convergence rates are obtained by RBF, which, in some cases, reaches sensibly higher convergences rates.

\section{Discussion and conclusion}

In this paper the reconstruction of the first and second derivatives of the finite element solution has been addressed with particular focus on mechanical problems. For these problems, the solution of finite element problems is the displacement field and the most relevant derivatives of this field are the stress field (related to the first derivative) and the stress gradient field (related to the second derivative).

Three local reconstruction techniques have been considered for stress field reconstruction and four for the stress gradient. These techniques have been evaluated by two examples of practical interest (one two dimensional and one three dimensional). For these examples the analytical solution are available and the reconstruction error can be evaluated. The reconstruction error has been evaluated in a region inside the model domain and in a region on the border of the domain for four different kinds of elements (two linear and two quadratic both in $2 \mathrm{D}$ and in $3 \mathrm{D})$.

The two examples have shown the different performances of the considered reconstructing techniques when non polynomial stress and stress gradient fields are considered both inside the computational domain and on its border.

Among the usual reconstruction techniques, the $\mathrm{ZZ}$ patch recovery for stress and the modified version of the double ZZ patch recovery for stress gradients have shown the best global performances. From a computational point of view, these techniques are the most demanding in terms of algorithm complexity and have an high computational time. In particular, the patch search can be complicated, especially for three dimensional quadratic tetrahedron. A series of special cases has to be considered and different search algorithms have to be employed. The computational time is related to the inversion of the coefficient matrix.

The polynomial preserving and the double polynomial preserving reconstructions have shown similar performances of the corresponding ZZ techniques in almost all of the considered cases. From a computational point of view, the search algorithm is simpler, not requiring to distinguish between different cases. The computational time is generally higher as the order of the considered polynomials is higher, thus requiring more coefficients to be computed. The applicability of this method seems restricted only to elastic material models.

The nodal average technique for stress reconstruction is the simplest to implement and the faster in computation not requiring any matrix inversion. The performances of this technique are similar to the other two for internal nodes. For nodes on the boundary, a lower level of accuracy has been found.

Shape function based and stress field gradient analysis techniques for stress gradient reconstruction are both based on the nodal stress field reconstructed by the nodal average. For this reason, both technique show poor performances in the reconstruction of the stress gradient on the border of the domain. In some cases, for linear elements these technique do not converge. From a computational point of view, the shape function based technique is the simplest to implement and quite fast in computation not requiring any matrix inversion. Moreover, this technique uses the information of only one element at the time, thus not requiring any search algorithm. The stress field gradient analysis is based on just one layer of neighbour nodes and the matrix to be inverted is usually small. The search algorithm is simpler than the other two cases and also the computational time is lower.

The radial basis function network approach has shown potentialities to well reconstruct both stress and stress gradient fields with performances comparable or better, with respect to the most accurate reconstruction techniques in most of the considered cases. The implementation of the method is relative simple being the radial basis function network a well known technique and many algorithms are readily available for its implementation. The memory request of this approach is very high because in the training phase of the neural network a dense matrix containing the displacements of all nodes has to be inverted. In most cases, if the whole model is fed to the neural network, the memory requested is larger than the memory needed to solve the 

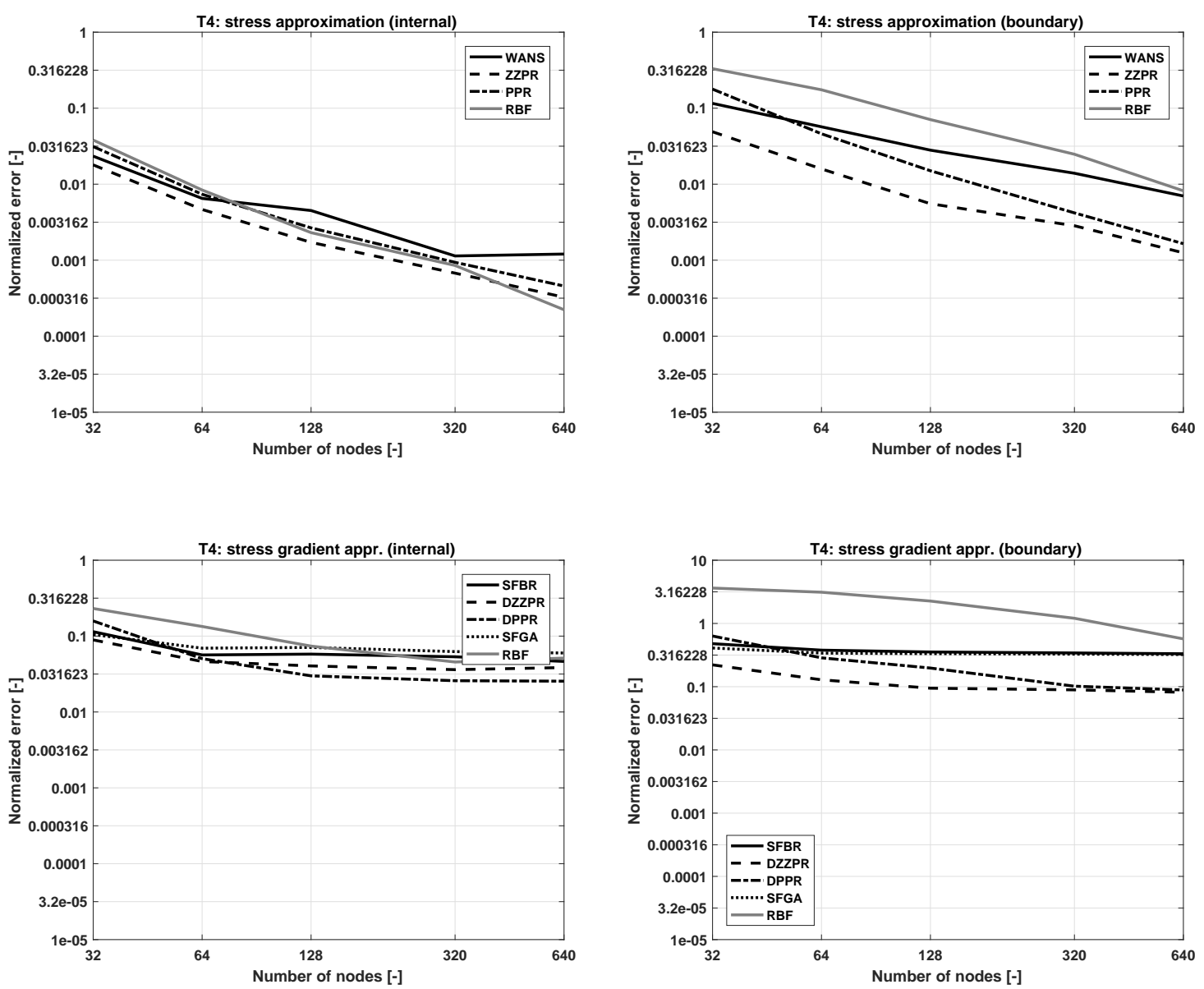

Fig. 13 T4 elements: normalized mean error (eq. 18) as function of the number of nodes on the considered line. Top: stress estimation (Left: path B of fig. 11, Right path A of fig. 11). Bottom: stress gradient estimation (Left: path B of fig. 11, Right path A of fig. 11).

Table 3 Convergence rates for the considered reconstruction techniques for the rotating thick cylinder example. Paths A and B definition in Fig. 11

Internal nodes (path B)

\begin{tabular}{|c|c|c|c|c|c|c|c|c|c|}
\hline & \multicolumn{4}{|c|}{ Stress reconstruction } & \multicolumn{4}{c|}{ Stress gradient reconstruction } \\
\hline & WANS & ZZPR & PPR & RBF & SFBR & DZZPR & DPPR & SFGA & RBF \\
T4 & 1.01 & 1.30 & 1.38 & 1.64 & - & - & - & - & - \\
T10 & 1.77 & 2.17 & 1.47 & 2.26 & 1.04 & 1.44 & 0.76 & 1.08 & 1.64 \\
B8 & 1.82 & 1.82 & 1.96 & 2.40 & 1.81 & 1.81 & 1.30 & 1.81 & 2.37 \\
B20 & 1.83 & 3.51 & $\sim 2$ & 4.11 & 1.78 & 2.51 & $\sim 2$ & 0.96 & 4.40 \\
\hline
\end{tabular}

Border nodes (path A)

\begin{tabular}{|c|c|c|c|c|c|c|c|c|c|}
\hline & \multicolumn{3}{|c|}{ Stress reconstruction } & \multicolumn{4}{c|}{ Stress gradient reconstruction } \\
\hline & WANS & ZZPR & PPR & RBF & SFBR & DZZPR & DPPR & SFGA & RBF \\
T4 & 0.92 & 1.18 & 1.54 & 1.23 & - & - & - & - & - \\
T10 & 1.66 & 2.08 & 1.97 & 2.09 & 0.81 & 1.32 & 1.17 & 0.85 & 1.74 \\
B8 & 0.88 & 1.88 & 1.49 & 3.23 & 0.10 & 1.11 & 0.62 & 0.10 & 2.31 \\
B20 & 1.69 & 2.37 & 1.70 & 2.53 & 0.78 & 1.26 & 0.72 & 0.96 & 1.87 \\
\hline
\end{tabular}



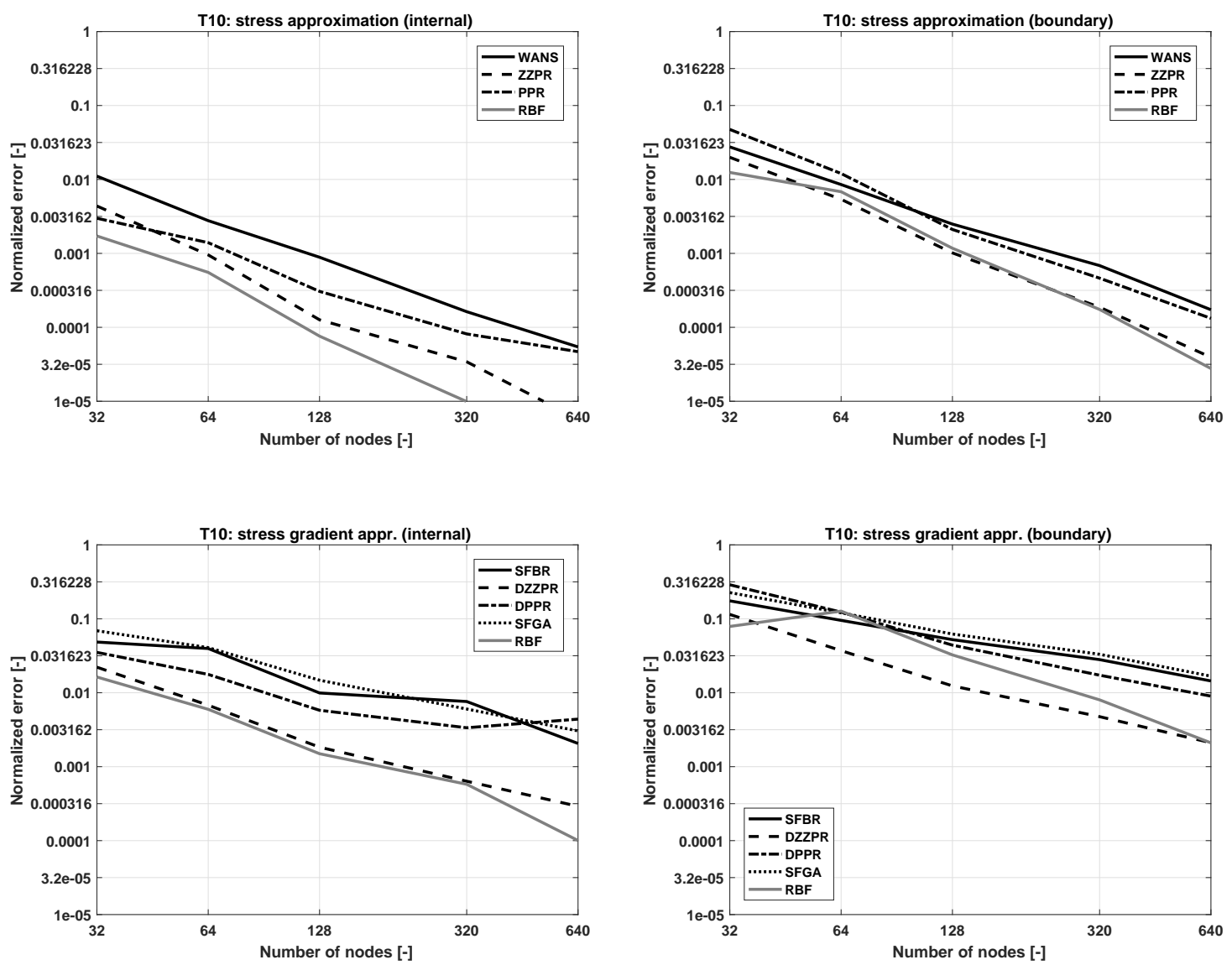

Fig. 14 T10 elements: normalized mean error (eq. 18) as function of the number of nodes on the considered line. Top: stress estimation (Left: path B of fig. 11, Right path A of fig. 11). Bottom: stress gradient estimation (Left: path B of fig. 11, Right path A of fig. 11).

FE model itself. To circumvent memory limitations, the model can be divided into few relatively large regions to be fed to the neural network one at the time. The computational time is function of the actual number of nodes fed to the neural network, but for reasonable dimensions of the considered regions is comparable with the other reconstruction techniques. In case the shape paramter is not chosen a priory, but an optimization process is required, the computational time can increment considerably.

\section{Conflict of interest}

The authors declare that they don't have conflict of interests.

\section{References}

1. ALTAIR (2014) Optistruct user's guide
2. Benedetti A, de Miranda S, Ubertini F (2006) A posteriori error estimation based on the superconvergent Recovery by Compatibility in Patches. International Journal for Numerical Methods in Engineering 67:108-131

3. Björck $\AA$ (1996) Numerical methods for least squares problems. Society for Industrial and Applied Mathematics, Philadelphia U.S.A.

4. Blacker T, Belytschko T (1994) Superconvergent patch recovery with equilibrium and conjoint interpolant enhancements. International Journal for Numerical Methods in Engineering 37(3):517536, DOI 10.1002/nme.1620370309, URL http://doi.wiley.com/10.1002/nme.1620370309

5. Booromand B, Zienkievicz OC (1997) Recovery by equilibrium in patches (REP). Int J Numer Methods Eng 40:137-164 

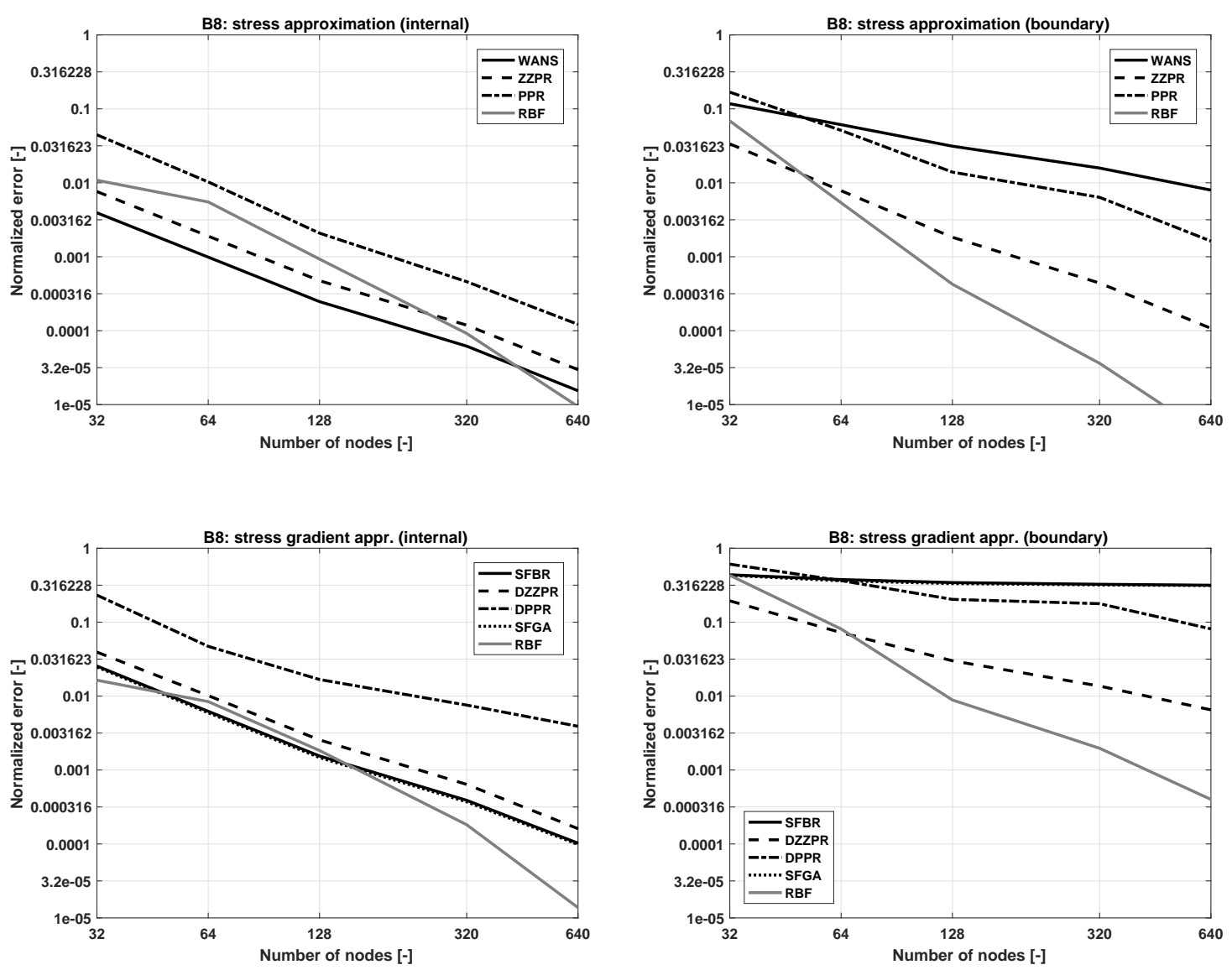

Fig. 15 B8 elements: normalized mean error (eq. 18) as function of the number of nodes on the considered line. Top: stress estimation (Left: path B of fig. 11, Right path A of fig. 11). Bottom: stress gradient estimation (Left: path B of fig. 11, Right path A of fig. 11).

6. Broomhead DS, Lowe D (1988) Multivariable Functional Interpolation and Adaptive Networks. Complex Systems 2 pp 321-355

7. Chen J, Li CJ (2014) A quadrilateral spline element for couple stress/strain gradient elasticity. Computers \& Structures 138:133-141

8. Duy NM, Cong TT (2003) Approximation of function and its derivatives using radial basis function networks. Applied Mathematical Modelling 27(3):197-220, DOI 10.1016/S0307904X(02)00101-4

9. Ettehad M, Abu Al-Rub RK (2015) On the numerical implementation of the higher-order strain gradient-dependent plasticity theory and its nonclassical boundary conditions. Finite Elements in Analysis and Design 93:50-59

10. Ferreira AJ, Roque CM, Carrera E, Cinefra M, Polit O (2013) Bending and vibration of lami- nated plates by a layerwise formulation and collocation with radial basis functions. Mechanics of Advanced Materials and Structures 20(8):624-637, DOI 10.1080/15376494.2011.643282

11. Gan X, Akin JE (2014) Super-convergent second derivative recovery for lower-order strain gradient plasticity. Computers \& Structures 135:118-127

12. Grätsch T, Bathe KJ (2005) A posteriori error estimation techniques in practical finite element analysis. Computers and Structures 72:235-265

13. Guo H, Zhang Z, Zhao R (2014) Hessian Recovery for Finite Element Methods. arXiv:14063108 pp 119

14. Han CS, Wriggers P (2000) An h-adaptive method for elastoplastic shell problems. Computer Methods in Applied Mechanics and Engineering 189:651-671

15. Han CS, Ma A, Roters F, Raabe D (2007) A finite element approach with patch projection for 

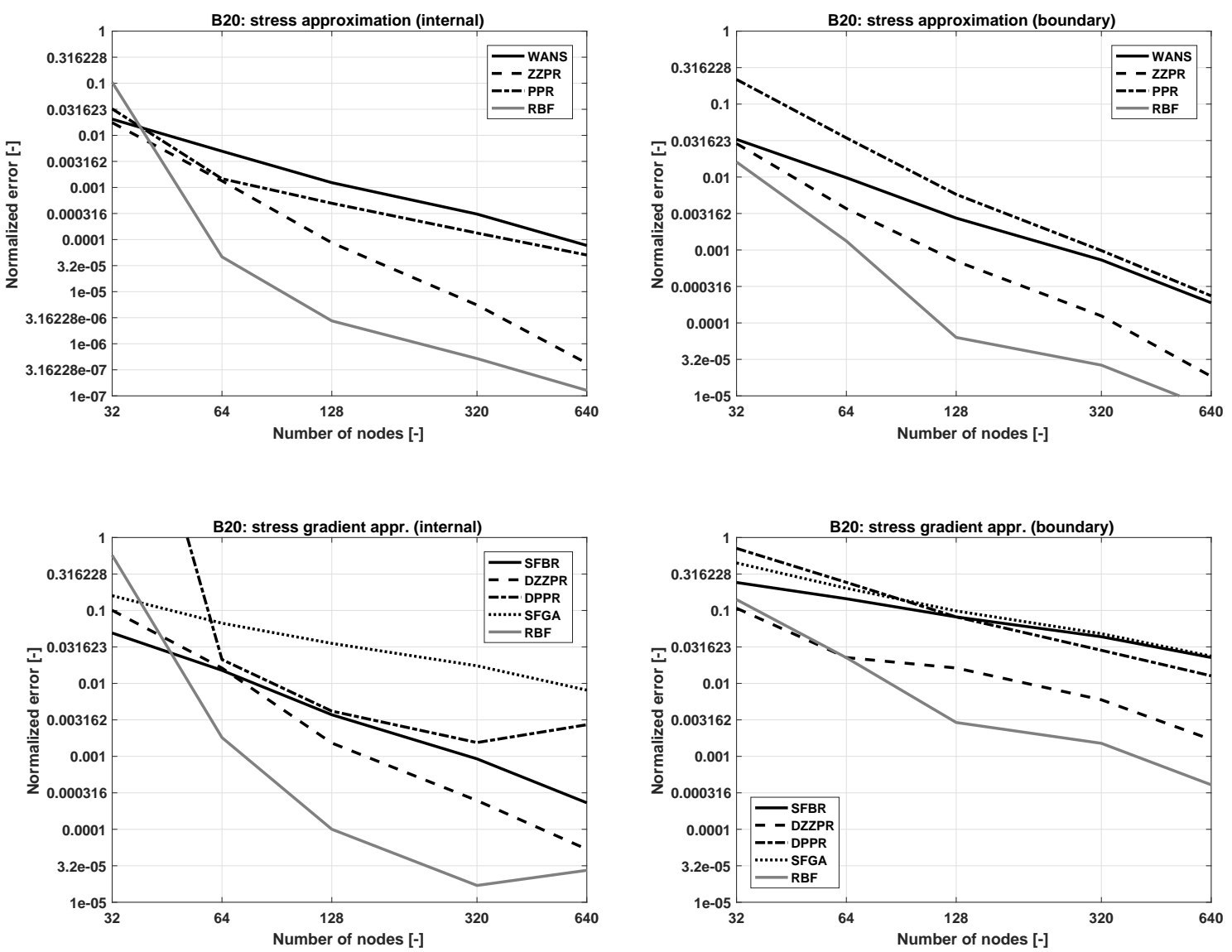

Fig. 16 B20 elements: normalized mean error (eq. 18) as function of the number of nodes on the considered line. Top: stress estimation (Left: path B of fig. 11, Right path A of fig. 11). Bottom: stress gradient estimation (Left: path B of fig. 11, Right path A of fig. 11).

strain gradient plasticity formulations. International Journal of Plasticity 23:690-710

16. Haykin S (1999) Neural Networks : A Comprehensive Foundation. Prentice-Hall

17. Kahrobaiyan MH, Asghari M, Ahmadian MT (2013) Strain gradient beam element. Finite Elements in Analysis and Design 68:63-75

18. Karageorghis A, Chen CS, Smyrlis YS (2007) A matrix decomposition RBF algorithm: Approximation of functions and their derivatives. Applied Numerical Mathematics 57(3):304-319, DOI 10.1016/j.apnum.2006.03.028

19. Kim J, Bathe KJ (2013) The finite element method enriched by interpolation covers. Computers and Structures 116:35-49

20. Lee T, Park HC, Lee SW (1997) A Superconvergent stress recovery technique with equilibrium constraint. Numerical Methods in Engineer- ing 40:1139-1160

21. Li B, Zhang Z (1999) Analysis of a class of superconvergence patch recovery techniques for linear and bilinear finite elements. Numerical Methods for Partial Differential Equations 15:151-167

22. Lin R, Zhan Z (2008) Natural superconvergent points in three-dimensional finite elements. SIAM Journal of numerical Analysis 46:1281-1297

23. LMS SIEMENS (2015) LMS Virtual Lab Durability Theory Manual

24. Lo SH, Lee CK (1998) On using different recovery procedures for the construction of smoothed stress in finite element method. International Journal for Numerical Methods in Engineering 43:1223-1252

25. Martinez-Paneda E, del Busto S, Niordson CF, Betegon C (2016) Strain gradient plasticity modeling of hydrogen diffusion to the crack tip. International Journal of Hydrogen Energy pp 10265-10274 
26. Maturi DA, Ferreira AJ, Zenkour AM, Mashat DS (2013) Analysis of laminated shells by Murakami's Zig-Zag theory and radial basis functions collocation. Journal of Applied Mathematics 2013:1-14, DOI 10.1155/2013/123465

27. Matusov JB (1995) Multicriteria Optimisation and Engineering. Chapman \& Hall, New York

28. Mcdonald DB, Grantham WJ, Tabor WL, Murphy MJ (2007) Global and local optimization using radial basis function response surface models. Applied Mathematical Modelling 31(10):20952110, DOI 10.1016/j.apm.2006.08.008

29. MSC (2014) Nastran 2014 Linear Static Analysis User's Guide

30. Naga A, Zhang Z (2004) A posteriori error estimates based on the polynomial preserving recovery. SIAM Journal Numeric Analysis 42:1780-1800

31. Papadopoulos IV (1996) Invariant formulation of a gradient dependent multiaxial high-cycle fatigue criterion. Engineering Fracture Mechanics 55:513528

32. Payen DJ, Bathe KJ (2012) A stress improvement procedure. Computers and Structures 112-113:311326

33. Picasso M, Alauzet F, Borouchaki H, George PL (2011) A Numerical Study of Some Hessian Recovery Techniques on Isotropic and Anisotropic Meshes. SIAM Journal for Scientific Computing 33:1058-1076

34. Polizzotto C (2016) Variational formulations and extra boundary conditions within stress gradient elasticity theory with extensions to beam and plate models. International Journal of Solids and Structures 80:405-419, DOI 10.1016/j.ijsolstr.2015.09.015

35. Pouliot B, Fortin M, Fortin A, Chamberland E (2013) On a new edge-based gradient recovery technique. International Journal for Numerical Methods in Engineering 93:52-65

36. Rankovic V, Radulovic J (2011) Prediction of magnetic field near power lines by normalized radial basis function network. Advances in Engineering Software 42:934-938

37. Ródenas JJ, Tur M, Fuenmayor FJ, Vercher A (2007) Improvement of the superconvergent patch recovery technique by the use of constraint equations: the SPR-C technique. International Journal for Numerical Methods in Engineering 70(6):705-727, DOI 10.1002/nme.1903, URL http://doi.wiley.com/10.1002/nme.1903

38. Schneider R (2013) A Review of Anisotropic Refinement Methods for Triangular Meshes in FEM in Advanced Finite Element Methods and Ap- plications eds.Thomas Apel and Olaf Steinbach, Springer, Berlin

39. Simulia (2014) ABAQUS Analysis User's Guide, ver. 6.14

40. Soh AK, Wanji C (2001) Finite element formulations of strain gradient theory for microstructures and the $\$ \mathrm{C}^{\wedge}\{0-1\} \$$ patch test. International Journal of Plasticity 50:1369-1388

41. Stein E, Ohnimus S (1997) Equilibrium method for postprocessing and error estimation in the finite element method. Computer Assisted Mechanics and Engineering Sciences 4:645-666

42. Ubertini F (2004) Patch recovery based on complementary energy. Int J Numer Methods Eng 59:1501-1538

43. Vallet MG, Manole CM, Dompierre J, Dufour S, Guibault F (2007) Numerical comparison of some Hessian recovery techniques. International Journal for Numerical Methods in Engineering 72:987-1007

44. Varias AG, Massih AR (2000) Simulation of hydrogen embrittlement in zirconium alloys under stress and temperature gradients. Journal of Nuclear Materials pp 273-285

45. Wang BP (2004) Parameter optimization in multiquadric response surface approximations. Structural and Multidisciplinary Optimization 26(34):219-223, DOI 10.1007/s00158-003-0341-4, URL http://dx.doi.org/10.1007/s00158-003-0341-4

46. Wang JG, Liu GR (2002) A point interpolation meshless method based on radial basis functions. Int J Numer Methods Eng 54:1623-1648

47. Wang JG, Liu GR (2002) On the optimal shape parameters of radial basis functions used for 2-D meshless methods. Computer methods in applied mechanics and engineering 191:2611-2630

48. Wilberg NE (1997) Superconvergent Patch Recovery - a key to quality assessed FE solutions. Advances in Engineering Software 28:85-95

49. Xing J, Zheng G (2015) Stress Field Gradient Analysis Technique Using Lower-Order $\$$ C_0 $\$$ Elements. Mathematical Problems in Engineering 2015:1-12, DOI 10.1155/2015/457046

50. Zervos A, Papanastasiou P, Vardoulakis I (2001) A finite element displacement formulation for gradient elastoplasticity. International Journal of Plasticity 50:1369-1388

51. Zervos A, Papanicolopulos SA, Vardoulakis I (2009) Two finite element discretizations for gradient elasticity. Journal of Engineering Mechanics (ASCE) 135:203-213

52. Zhan Z, Lin R (2003) Ultraconvergence of ZZ patch recovery at mesh symmetry points. Numerische Mathematik 95:781-801 
53. Zhang Z (2000) Ultraconvergence of the patch recovery technique. Mathematics of Computation 69:141-158

54. Zhang Z, Naga A (2005) A new finite element gradient recovery method: superconvergence property. SIAM Journal for Scientific Computing 26:11921213

55. Zienkiewicz O, Zhu JZ (1992) The superconvergence patch recovery and a posteriori error estimates. Part 1: The recovery technique. International Journal for Numerical Methods in Engineering 33:1331-1364

56. Zienkiewicz O, Zhu JZ (1992) The superconvergence patch recovery and a posteriori error estimates. Part 2: error estimates and adaptivity. International Journal for Numerical Methods in Engineering 33:1365-1382

57. Zienkiewicz OC, Taylor RL (2000) The Finite Element Method: the Basis, $\$ 5^{\wedge}\{$ th $\} \$$ edition. Butterwoth Heinemann, Oxford

\section{A Expressions of the monomials for the $\mathrm{ZZ}$ patch recovery}

In this Annex the expression of the monomials functions used for the ZZ patch recovery of Sect. 2.2 are reported. The monomial functions have the same expression of the shape functions of the elements for all elements but the bilinear quadrilateral $(Q 4)$. For this element, the patches are respectively of 4 elements with just one sampling point for element. The number o sampling points corresponds exactly to the number of unknowns of a bilinear function in 2D. To avoid numerical problems of ill-conditioned coefficient matrices and numerical instabilities, for this element, a function with less terms than the shape functions is used.

Table 4 reports the monomial expressions for the considered elements.

\section{B Expressions of the monomials for the meshless gradient recovery}

In this Annex the expression of the monomials functions used for the polynomial preserving recovery of Sect. 2.3 are reported. Table 5 reports the monomial expressions for the considered elements along with the minimum number of considered nodes. The minimum number of nodes is chosen as to guarantee at least two levels of surrounding elements. 
Table 4 Monomials functions for ZZ patch recovery (Sect. 2.2)

\begin{tabular}{|c|c|c|c|}
\hline $\begin{array}{l}\text { Element } \\
\text { type }\end{array}$ & Dimension & Order & Monomials function \\
\hline T3 & $2 \mathrm{D}$ & linear & $\zeta_{1}=\left[\begin{array}{lll}1 & x & y\end{array}\right]$ \\
\hline Q4 & $2 \mathrm{D}$ & linear & $\zeta_{1}=\left[\begin{array}{lll}1 & x & y\end{array}\right]$ \\
\hline T6 & $2 \mathrm{D}$ & quad. & 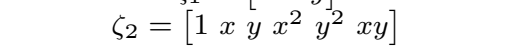 \\
\hline Q8 & $2 \mathrm{D}$ & quad. & 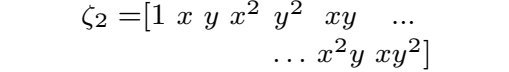 \\
\hline $\mathrm{T} 4$ & $3 \mathrm{D}$ & linear & $\zeta_{1}=\left[\begin{array}{llll}1 & x & y & z\end{array}\right]$ \\
\hline B8 & $3 \mathrm{D}$ & linear & $\zeta_{1}=\left[\begin{array}{lllllll}1 & x & y & z & x y & x z & y z\end{array}\right]$ \\
\hline $\mathrm{T} 10$ & $3 \mathrm{D}$ & quad. & 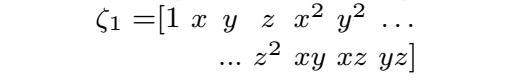 \\
\hline B20 & $3 \mathrm{D}$ & quad. & 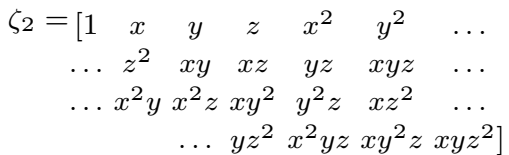 \\
\hline
\end{tabular}

Table 5 Monomials functions for polynomial preserving patch recovery (Sect. 2.3)

\begin{tabular}{|c|c|c|c|}
\hline $\begin{array}{l}\text { Element } \\
\text { typev }\end{array}$ & Dimension & $\begin{array}{l}\text { Min. node } \\
\text { number }\end{array}$ & Monomials function \\
\hline T3, Q4 & $2 \mathrm{D}$ & 15 & 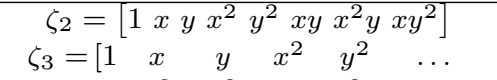 \\
\hline T6, Q8 & $2 \mathrm{D}$ & 25 & 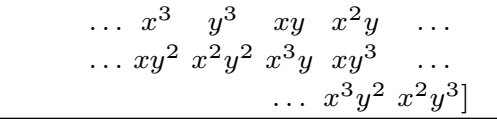 \\
\hline T4, B8 & $3 \mathrm{D}$ & 30 & 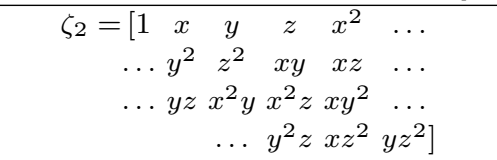 \\
\hline $\mathrm{T} 10, \mathrm{~B} 20$ & $3 \mathrm{D}$ & 75 & 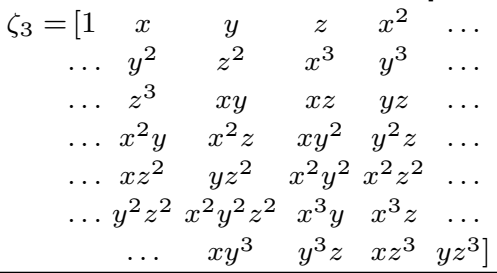 \\
\hline
\end{tabular}

